\title{
NEWLY QUENCHED GALAXIES AS THE CAUSE FOR THE APPARENT EVOLUTION IN AVERAGE SIZE OF THE POPULATION
}

\author{
C. M. Carollo ${ }^{1}$, T. J. Bschorr ${ }^{1}$, A. Renzini ${ }^{2}$, S. J. Lilly ${ }^{1}$, P. Capak $^{3}$, A. Cibinel $^{1}$, O. Ilbert ${ }^{4}$, M. Onodera $^{1}$, \\ N. Scoville ${ }^{5}$, E. Cameron ${ }^{1}$, B. Mobasher ${ }^{6}$, D. Sanders $^{7}$, And Y. TAniguchi $^{8}$ \\ ${ }^{1}$ Institute for Astronomy, Swiss Federal Institute of Technology (ETH Zurich), CH-8093 Zurich, Switzerland; marcella@ phys.ethz.ch \\ ${ }^{2}$ Istituto Nazionale di Astrofisica, Osservatorio Astronomico di Padova, Vicolo dell'Osservatorio 5, I-35121 Padova, Italy \\ ${ }^{3}$ Spitzer Science Center, California Institute of Technology, 1200 East California Boulevard, Pasadena, CA 91125, USA \\ ${ }^{4}$ Laboratoire d'Astrophysique de Marseille, 38 rue Frederic Joliot Curie, F-13388 Marseille, France \\ ${ }^{5}$ California Institute of Technology, MC 105-24, 1200 East California Boulevard, Pasadena, CA 91125, USA \\ ${ }^{6}$ Department of Physics and Astronomy, University of California, Riverside, CA 92508, USA \\ ${ }^{7}$ Institute for Astronomy, University of Hawaii, 2680 Woodlawn Dr, Honolulu, HI 96822, USA \\ ${ }^{8}$ Research Center for Space and Cosmic Evolution, Ehime University, Bunkyo-cho 2-5, Matsuyama 790-8577, Japan \\ Received 2013 February 18; accepted 2013 June 7; published 2013 August 1
}

\begin{abstract}
We use the large COSMOS sample of galaxies to study in an internally self-consistent way the change in the number densities of quenched early-type galaxies (Q-ETGs) of a given size over the redshift interval $0.2<z<1$ in order to study the claimed size evolution of these galaxies. In a stellar mass bin at $10^{10.5}<M_{\text {galaxy }}<10^{11} M_{\odot}$, we see no change in the number density of compact Q-ETGs over this redshift range, while in a higher mass bin at $>10^{11} M_{\odot}$, where we would expect merging to be more significant, we find a small decrease, by $\sim 30 \%$. In both mass bins, the increase of the median sizes of Q-ETGs with time is primarily caused by the addition to the size function of larger and more diffuse Q-ETGs. At all masses, compact Q-ETGs become systematically redder toward later epochs, with a $(U-V)$ color difference which is consistent with a passive evolution of their stellar populations, indicating that they are a stable population that does not appreciably evolve in size. We find furthermore, at all epochs, that the larger Q-ETGs (at least in the lower mass bin) have average rest-frame colors that are systematically bluer than those of the more compact Q-ETGs, suggesting that the former are indeed younger than the latter. The idea that new, large, Q-ETGs are responsible for the observed growth in the median size of the population at a given mass is also supported by analysis of the sizes and number of the star-forming galaxies that are expected to be the progenitors of the new Q-ETGs over the same period. In the low mass bin, the new Q-ETGs appear to have $\sim 30 \%$ smaller half-light radii than their star-forming progenitors. This is likely due to the fading of their disks after they cease star formation. Comparison with higher redshifts shows that the median size of newly quenched galaxies roughly scales, at constant mass, as $(1+z)^{-1}$. We conclude that the dominant cause of the size evolution seen in the Q-ETG population is that the average sizes and thus stellar densities of individual Q-ETGs roughly scale with the average density of the universe at the time when they were quenched, and that subsequent size changes in individual objects, through merging or other processes, are of secondary importance, especially at masses below $10^{11} M_{\odot}$.
\end{abstract}

Key words: galaxies: evolution - galaxies: formation - galaxies: fundamental parameters - galaxies: structure

\section{INTRODUCTION}

The evolution of the median size (i.e., the half-light radius $\left.r_{1 / 2}\right)$ of the population of massive $\left(M_{\text {Galaxy }}>10^{10} M_{\odot}\right)$ quenched early-type galaxies (Q-ETGs) at given stellar mass has been widely highlighted in recent years (e.g., Daddi et al. 2005; Trujillo et al. 2007; McGrath et al. 2008; van Dokkum et al. 2008; Cassata et al. 2011; Szomoru et al. 2011; Barro et al. 2013; Dullo \& Graham 2013; Newman et al. 2012; Poggianti et al. 2013; Shankar et al. 2013, just to cite a few). The size of the effect is quite large, with a decrease in median $r_{1 / 2}$ with increasing redshift $\propto(1+z)^{-1}$; in coarse terms, this implies that, at a given stellar mass, the median half-light radius of Q-ETGs is about a factor of $\sim 2-3$ smaller at $z \sim 2$ than locally, corresponding to an increase of over an order of magnitude in the median mean stellar density within the half-light radius of galaxies.

The wealth of studies quoted above have used a variety of imaging data taken from space and from the ground, at different wavelengths, and have focused on galaxy populations at different redshifts. Quite naturally, there has been some debate as to whether obvious observational biases might have affected the results, such as the possible loss in the noise of outer, low surface brightness parts of the galaxies, or the possible effects of color gradients (e.g., Daddi et al. 2005; Mancini et al. 2010). Younger stellar populations in the cores of galaxies could result in smaller sizes in the rest-frame ultraviolet, where the sizes are often measured, than at the longer wavelengths, which better sample the stellar mass distribution.

Many of the studies cited above have attempted to deal with these uncertainties (e.g., Mancini et al. 2010; Szomoru et al. 2011), and there is now a reasonable consensus that there is a real effect to be explained. For example, there appear to be no strong color gradients in high- $z$ Q-ETG in those cases in which both rest-frame UV and optical imaging are available (e.g., Toft et al. 2007; Guo et al. 2011). Thus, there is now general consensus that indeed the median size of Q-ETGs is substantially smaller at high redshifts, though apparently normally sized Q-ETGs coexist with compact ones, especially among the most massive galaxies (e.g., Saracco et al. 2010; Mancini et al. 2010; see also Onodera et al. 2010 for a similar conclusion concerning the velocity dispersion of Q-ETGs at $z=2$ ).

With some exceptions (e.g., Valentinuzzi et al. 2010; Cassata et al. 2011; Newman et al. 2012; Poggianti et al. 2013), this trend has been often entirely ascribed to the physical growth of individual galaxies. Rather than a puff-up mechanism, 
decreasing the central stellar density of Q-ETGs, the favored picture has been one in which Q-ETGs maintain a nearly constant mass within their innermost few kiloparsecs, and gradually grow inside-out, building up extended stellar envelopes/halos around such compact, dense cores (e.g., Cimatti et al. 2008; Hopkins et al. 2009, 2010; Taylor et al. 2010; Feldmann et al. 2010; Szomoru et al. 2011). Accretion of small satellites in minor gas-poor mergers has been widely entertained as the leading mechanism to grow these stellar envelops (e.g., Naab et al. 2009; Hopkins et al. 2009; Feldmann et al. 2010; Nipoti et al. 2009; Oser et al. 2012) and thus increase the radius of individual high- $z$ compact Q-ETGs, until they reach their final $z=0$ dimension.

Yet, this may be only part of the story, and possibly a minor one. First, it is now solidly established that the population of Q-ETGs has undergone a strong increase in comoving number density between $z \sim 2$ and the present epoch (e.g., Williams et al. 2009; Ilbert et al. 2010, 2013; Domínguez Sánchez et al. 2011). The mass functions of different galaxy populations in Ilbert et al. (2010, 2013), based on high-quality photometric redshifts in the COSMOS field (Scoville et al. 2007), indicate that the number density of quiescent galaxies has increased by a factor of $\sim 2$ since $z \sim 1$, and by a factor of at least 10 since $z \sim 2$. These observed number density growth factors for Q-ETGs match those expected by applying a simple continuity equation to the time evolution of the actively star-forming galaxy population (Peng et al. 2010, 2012).

In addition, the analysis of Sloan Digital Sky Survey (SDSS; York et al. 2007) mass functions shows that typical passive galaxies with $M<10^{11} M_{\odot}$ can only have increased their masses by around $20 \%$ (and definitely less than $40 \%$ ) after quenching (Peng et al. 2010). Analysis of the mass functions of SDSS central and satellite galaxies in Peng et al. (2012) refines these estimates to an average post-quenching mass increase of $\sim 25 \%$ for typical central galaxies, and a negligible increase for satellite galaxies. These constraints strongly limit the amount of merging that may be available to increase the sizes of galaxies. This, together with the evolution of the number density, can explain why the minor dry merging scenario falls somewhat short from quantitatively accounting the observed size growth since $z \sim 2$ (e.g., Oser et al. 2012).

It seems therefore quite likely that the advocated afterquenching growth of individual Q-ETGs contributes only modestly to the observed secular increase of the median size of the QETG population; given the large increase of the number density of these systems since $z \sim 2$, it is plausible that another effect may dominate, namely, quenching of star formation in actively star-forming galaxies that keeps producing, at later epochs, new Q-ETGs with larger size than those of galaxies quenched at earlier epochs, as partly advocated on heuristic evidence by, e.g., Valentinuzzi et al. (2010), Cassata et al. (2011), Newman et al. (2012), and Poggianti et al. (2013). The addition, at progressively lower redshifts, of progressively larger Q-ETGs will progressively lower the relative fraction of the more compact galaxies relative to the total Q-ETG population, and thus produce an upward evolution of the size-mass relation. There are good reasons, in an expanding universe that grows structure hierarchically, to entertain the notion that later-appearing Q-ETGs will be larger and thus have lower stellar densities than galaxies of similar stellar mass that are quenched at earlier epochs (e.g., Covington et al. 2011). The apparent disappearance of compact Q-ETGs at later epochs may thus be a false reading of a reality in which earlier populations of denser Q-ETGs remain relatively stable in terms of numbers through cosmic time, but become less and less important, in relative number, at later and later epochs.

An important question to answer is thus how the number density of compact Q-ETGs evolves from high redshifts all the way down to the local universe. Searches for local analogs to the compact, massive Q-ETGs observed at $z \sim 2$ have been undertaken to answer this question, and have given conflicting results. Specifically, for compact Q-ETGs in massive galaxy clusters, Valentinuzzi et al. (2010) have reported evidence for little or no evolution between $z \sim 0.7$ and $z \sim 0.04$ in this population. Comparing to SDSS DR7 (Abazajian et al. 2009), other studies have also argued for not much evolution in the number density of compact Q-ETGs between $z \sim 1.5$ and the present (e.g., Saracco et al. 2010). Other SDSS studies have however reported a drop of at least a factor $\sim 20$ between $z \sim 1.6$ and $z=0.1$ (Cassata et al. 2011) or even more dramatic than this (e.g., Taylor et al. 2010). Also, Szomoru et al. (2011) find that the minimum growth in size required to reconcile the size distribution of quenched galaxies at $z \sim 2$ with that of their counterparts at $z=0$ is a factor $\sim 2$ smaller than the total median size growth observed in the same redshift interval.

Some of the difference between the apparently conflicting results may in fact stem from compactness having been quantitatively defined in different ways by different authors, e.g., either in physical units, or relative to the average size-stellar mass relation for local Q-ETGs. Quite often, the fraction of compact Q-ETGs is considered, as opposed to their number density. Other aspects of the analyses need, however, to come under scrutiny to reconcile such widely diverse results. For example, Taylor et al. (2010), Cassata et al. (2011), and Szomoru et al. (2011) use published SDSS sizes as the comparison standard at $z=0$. This is risky, as the input photometric catalogs may have missed compact galaxies through an imperfect statistical star-galaxy separation (Scranton et al. 2002). Furthermore, as shown by Cibinel et al. (2012) on the galaxy sample of the Zurich Environmental Survey (ZENS; Carollo et al. 2012), galaxy sizes smaller than the seeing point-spread function (PSF) are not reliably recovered from ground-based imaging data. The generally poor PSF (FWHM well above $\sim 1^{\prime \prime}$ ) of the SDSS images casts doubts therefore as to whether the published SDSS galaxy catalogs are adequate for this purpose. Particularly suggestive is the number density evolution of compact galaxies presented by Cassata et al. (2011), which, based on the self-consistent analysis of GOODS images (Giavalisco et al. 2004) is rather flat from $z \sim 2.5$ down to $z \sim 0.5$, and dramatically drops since $z \sim 0.5$, due to the comparison of the GOODS-based data point at $z=0.5$ with the SDSS point at $z=0$. Furthermore, the analysis of the number densities in Cassata et al. sums up all galaxies above $10^{10} M_{\odot}$, and thus misses possible differential evolution with stellar mass.

The present paper seeks to explore this issue in a carefully controlled fashion by examining, in the redshift range $0.2<$ $z<1$, the evolution at constant size (i.e., half-light radius) of the number densities of Q-ETGs, and of their plausible starforming progenitors. We perform our analysis in two bins of stellar mass in which the $I_{814 W}<24$ COSMOS sample is complete up to $z=1$, i.e., $10^{10.5}-10^{11} M_{\odot}$ and $>10^{11} M_{\odot}$; these two bins straddle across the nearly redshift-invariant characteristic mass $M^{*} \sim 10^{11} M_{\odot}$ in the Schechter (1976) fit to the mass function of galaxies (e.g., Peng et al. 2012, and references therein), enabling us to search for differential effects above and below this mass scale. A strength of our analysis is the self-consistent use of data from a single survey, 
i.e., COSMOS, thereby avoiding basing our conclusions on comparisons between inhomogeneous samples, and in particular relying on the SDSS data for the low-redshift reference sample. The COSMOS field is ideal for this study, being unique in having both exquisite photometric redshift estimates for a very large number of galaxies, based on deep multi-band photometry, and high-resolution F814W (I-band) Hubble Space Telescope (HST) Advanced Camera for Surveys (ACS) images (Koekemoer et al. 2007) over a large, $\sim 1.8 \mathrm{deg}^{2}$ area. While limiting the redshift range between $z=1$ and $z=0.2$ restricts the evolutionary lever-arm relative to comparing higher redshift samples with SDSS catalogs, our approach has indeed the great advantage that the sizes of the galaxies can be measured in a uniform way from a single homogeneous data set of unparalleled statistical significance.

In our analysis, we use aperture measurements for determining the sizes of the galaxies from the ACS F814W images because of their higher stability relative to model fitting approaches when applied to the full morphological diversity of faint high-redshift galaxies. We fully calibrate, however, our size measurements against magnitude, size, ellipticity, and concentration biases, and show that, once both aperture and model-fit measurements are so calibrated, they agree well with each other, giving us confidence in their robustness.

In detail, the paper is organized as follows. Section 2 summarizes the data set and the basic measurements and describes the selection criteria for the final galaxy sample in detail. Section 3 presents the approach utilized to correct sizes and other structural parameters for systematic biases that affect raw measurements as a function of galaxy magnitude, size, concentration, and ellipticity. Section 4 presents the redshift evolution of the number densities at constant size and surface mass density (i.e., the size and surface mass density functions) for Q-ETGs, and thus our core result, i.e., the constancy of the compact Q-ETG population and the emergence of a newly quenched population of large ETGs over the $z=\rightarrow 0.2$ period. Section 5 presents the size and surface mass density functions for star-forming galaxies, and compares the number densities of the newly quenched galaxies with the number densities of star-forming galaxies, of similar masses and sizes, that are expected to quench in the $z=1 \rightarrow 0.2$ interval, based on a continuity-equation argument (Peng et al. 2010). This section also compares the rest-frame optical colors of compact and large-size populations of Q-ETGs, and shows that compact Q-ETGs become redder toward later epochs and, at least at masses below $10^{11} M_{\odot}$, they are also systematically redder at any epoch, and thus likely older, than corresponding large-size Q-ETGs. This reinforces the interpretation that the latter are the newcomers in the Q-ETG population, which are responsible for increasing the median size of ETGs toward later epochs, without substantial increase in size of individual galaxies. In Section 6 we conclude.

Four appendices present some details of our analysis. Specifically, Appendices A and B provide, respectively, extra information on the robustness of the measured star formation rates (SFR) and on the reliability of the corrections that we apply to the structural/size parameters; Appendix C highlights the general need to correct such latter parameters even for estimates based on surface brightness fitting algorithms which take into account the effects of the observational PSF; and Appendix D finally summarizes the procedure that we follow to derive quenching rates using the prescriptions of Peng et al. (2010).
A cosmological model with $\Omega_{\Lambda}=0.7, \Omega_{M}=0.3$, and $h=0.7$ is adopted, and magnitudes are quoted in the $\mathrm{AB}$ system throughout.

\section{THE DATA AND THE BASIC MEASUREMENTS}

\subsection{COSMOS}

We base our study on the COSMOS survey data set (Scoville et al. 2007), so as to capitalize on its high-quality compilation of multiwavelength imaging, including $H S T / \mathrm{ACS}$ data (Koekemoer et al. 2007), over a wide-area field. For the present analysis, we employ the ACS I-band source catalog of Leauthaud et al. (2007) containing 156,748 sources (102,007 of these tested to be reliable galaxies) down to a flux limit of $I_{814 \mathrm{~W}}=24 \mathrm{mag}$. The reliability of this catalog for galaxy photometry and morphological analysis was subsequently improved via extensive visual inspection and cleaning to remove artifacts, cosmic rays, and stars, and to identify deblending errors, leaving a total of 102,007 sources flagged as reliable galaxy detections.

For the purpose of estimating photometric redshifts and stellar masses, we match the ACS I-band source catalog against the Canada-France-Hawaii Telescope (CFHT) $i^{*}$-band (McCracken et al. 2010) and Subaru $i^{+}$-selected COSMOS Intermediate and Broad Band Photometry Catalog (Ilbert et al. 2009, 2010, hereafter the "I09 catalog"). This aperture-matched, photometric database, constructed with an updated implementation of the source detection procedure described in Capak et al. (2007), offers a large compilation of broad- and narrowband flux measurements in 3 arcsec apertures across 31 bands from UV-optical $(u)$ through to infrared $(8.0 \mu \mathrm{m})$. As described in Capak et al. (2007) the use of PSF-matched, aperture magnitudes allows for a single correction ${ }^{9}$ to total flux across all bands for each object; estimates of these corrections are pre-compiled in the I09 catalog and we adopt these for the present analysis. Two further filter-specific corrections to these total fluxes are then required prior to photometric redshift estimation: (1) a correction against foreground Galactic dust reddening, for which we employ the Schlegel et al. (1998) extinction maps with wavelengthdependent adjustment factor, $k_{\lambda} \times E(B-V)$, from Cardelli et al. (1989) and (2) a correction against known zero point offsets in the COSMOS photometry, for which we employ the estimates derived from our photometric redshift package ZEBRA $^{10}$ run in catalog-correction mode (Feldmann et al. 2006). These corrections are consistent with those published for the COSMOS photometry by Ilbert et al. (2009) and Capak et al. (2007); they are based on $\chi^{2}$-minimization of fitting errors between the bestfit spectral energy distribution (SED) template and observed fluxes of galaxies with known redshifts from the zCOSMOS 20k sample (Lilly et al. 2007, 2009; see Feldmann et al. 2006).

For book-keeping purposes, we note that our procedure for matching the HST/ACS I-band source catalog against the I09 multiwavelength photometry catalog (2,017,800 sources) using a 0.6 arcsec tolerance on the centroid offset yields a total of $94,908(93.0 \%)$ direct galaxy matches (i.e., unique galaxy-galaxy associations). A further 5267 (5.2\%) galaxies

\footnotetext{
9 The four Spitzer mid-IR IRAC bands (Sanders et al. 2007) are an exception to this rule as it was unfeasible to degrade the optical data to the much broader Spitzer PSF; rather, the compiled IRAC fluxes were measured in fixed apertures of 1.9 arcsec and are corrected to total by dividing out factors of $0.76,0.74,0.62$, and 0.58 at $3.6 \mu \mathrm{m}, 4.5 \mu \mathrm{m}, 5.6 \mu \mathrm{m}$, and $8.0 \mu \mathrm{m}$, respectively (cf. Ilbert et al. 2009).

${ }^{10}$ The Zurich Extragalactic Bayesian Redshift Analyzer (ZEBRA) code is available online at our Web site, http://www.astro.ethz.ch/research/Projects/ZEBRA.
} 
were identified as sharing their match in I09 with another object in the HST/ACS catalog; based on our visual inspection of a few hundred such systems drawn randomly from the sample, these second matches are typically neighboring "junk sources" or overdeblended fragments of the original matched galaxy. We thus treat as successful matches the 3614 of these 5267 duplicates for which the primary match has a smaller centroid offset against its I09 counterpart than the additional (junk) candidate match. Conversely, due to the relatively broad PSF of the ground-based imaging used in construction of the I09 catalog, only a small number $(16$, i.e., $\sim 0.02 \%$ ) of galaxies in the HST/ACS-based sample were matched against more than one possible I09 counterpart within our 0.6 tolerance; once again, after visual inspection of these few sources, we adopted as the valid matches the sources displaying the smallest offset between the HST and I09 centroids. A total of 98,538 galaxies were thus deemed successfully matched, leaving only $1816(1.8 \%)$ galaxies in the parent $H S T /$ ACS catalog unmatched to any object in I09.

One further issue to deal with in the so-obtained galaxy sample is the large degree of flux contamination from interloper objects within the 3 arcsec adopted aperture in the I09 catalog. A total of 13,025 (13\% of our successful matches) were flagged by Capak et al. (2007) and Ilbert et al. (2009) as suffering severe contamination from either brighter neighbors or from the diffraction spikes of overexposed stars in at least one of the Subaru $B_{J}, V_{J}, i^{+}$, or $z^{+}$filters. Using such flagged objects may introduce errors in our scientific analysis, and hence we exclude these systems from our study. The completeness of our final galaxy sample is consequently $~ 84 \%(85,513 / 102,007)$, contributing a level of uncertainty to the absolute normalization of the size and surface mass density $\Sigma_{\text {MAss }}$ functions at each epoch computed herein comparable to that induced by cosmic variance (Trenti \& Stiavelli 2008) in the COSMOS field (Oesch et al. 2010). We checked that the completeness of our galaxy sample does not vary markedly with either size (i.e., half-light radius) or concentration index, so we do not expect any size- or morphology-dependent biases in the presented analysis.

\subsection{Photometric Redshifts, Stellar Masses, and Star Formation Rates}

We estimate photometric redshifts for objects in our matched source catalog using our ZEBRA code (Feldmann et al. 2006). Calibration of the benchmark SED templates (Coleman et al. 1980; Kinney et al. 1996) employed in this analysis was achieved by comparison against a sample of $\sim 20,000$ galaxies with secure spectroscopic redshifts from the zCOSMOS survey (Lilly et al. 2007, 2009). Only 236 of the 85,513 input matched galaxies were found to be outliers (i.e., a $0.3 \%$ failure rate). By comparison against the zCOSMOS sample at $I_{814 \mathrm{~W}}<22.5 \mathrm{mag}$, we estimate a photometric redshift uncertainty of $\Delta(z) /(1+z) \sim$ $0.007(1+z)$ at this brightness level. The uncertainty for galaxies down to $I_{814 W}=24$ mag was estimated to be $0.012(1+z)$ by artificially dimming the photometry of the zCOSMOS reference sample. The statistical quality of the ZEBRA photo- $z$ 's is very similar to that of the COSMOS photo- $z$ catalog of Ilbert et al. (2009). The latter was used to further validate the robustness of our results toward systematics uncertainties in the photo- $z$ 's.

For each galaxy for which a photometric redshift estimate could be obtained, we further employed a non-public extension of ZEBRA (i.e., "ZEBRA+"; see Oesch et al. 2010) to estimate the corresponding SFRs and stellar masses based on synthetic SED fitting to 11 photometric broad bands, ranging from $3832 \AA$ $\left(u^{*}, \mathrm{CFHT}\right)$ to $4.5 \mu \mathrm{m}$ (Spitzer/IRAC channel 2). ${ }^{11}$ The SED library consists of a comprehensive set of star formation history models, i.e., exponentially declining SFRs spanning a range of metallicities from 0.05 to $2 Z_{\odot}$, decay timescales from $\tau \sim 0.05$ to $9 \mathrm{Gyr}$, and ages from 0.01 to $12 \mathrm{Gyr}$ (with a Bayesian prior to bound the latter at less than the age of the universe at any given redshift). The construction of this library was achieved via the Bruzual \& Charlot (2003) stellar population synthesis code, adopting a Chabrier initial mass function (Chabrier 2003). The impact of dust extinction is handled during template matching by allowing dust reddening (Calzetti et al. 2000) with the $E(B-V)$ value treated as a free parameter of the fit. Synthetic template matches were identified, and a stellar mass successfully derived, for all but 1088 of the 85,277 COSMOS galaxies with photometric redshifts (a $1.3 \%$ failure rate). Owing to the inherent degeneracies in the choice of stellar population template and dust-extinction model, which dominate the error budget in the present analysis (given the thorough characterization of the observational SEDs across our multiwavelength database), we estimate an uncertainty of $\sigma_{\log M} \approx 0.20$ dex on our model stellar masses.

For the purposes of separating star-forming from quenched galaxies, we adopt a subdivision at an SED-fit specific star formation rate (i.e., SFR per unit stellar mass, hereafter sSFR) of $10^{-11} \mathrm{yr}^{-1}$; this corresponds closely to the inverse age of the universe at $z \sim 0.3$, the midpoint of the low redshift bin used in our analysis. In Appendix A, we explain our choice to use SFRs based on SED fits rather than (available) IR/UV-derived estimates. In Figure 1 we show, on the restframe near-UV (NUV) $-J$ versus $R-J$ diagram, the distributions of quenched and star-forming classified galaxies in the $0.2<z<0.4$ and $0.8<z<1.0$ redshift bins, respectively; star-forming and quenched galaxies are known to effectively separate in different regions in this diagnostic plane (e.g., Williams et al. 2009; Brammer 2009; Bundy et al. 2010). Inspection of the figure offers confidence that our chosen cut in sSFR well separates star-forming from quenched galaxies in our sample.

\subsection{Morphological Classification}

The morphological classifications were derived with the Zurich Estimator of Structural Types Plus (ZEST+), an upgraded version of the ZEST approach described in detail in Scarlata et al. (2007a). ZEST+ implements well-tested, robust algorithms for measuring a variety of non-parametric indices, including concentration, asymmetry, clumpiness, and Gini and $\mathrm{M}_{20}$ coefficients for a quantitative structural analysis and morphological classification of faint distant galaxies (see also references in Scarlata et al. 2007a). The new version of the algorithm, $Z E S T+$, features several substantial improvements relative to ZEST in key computations, including a quality-controlled removal of contaminating sources through substitution of optimal sky-valued pixels, a more robust identification of the galaxy centers, important especially for computations of asymmetry and concentration parameters, and a more robust calculation of the sources' Petrosian radii, unaffected by noise and contaminations by nearby sources. ZEST+ also implements a support vector machine (SVM) approach to estimate galaxy morphologies, in addition to the principal component analysis (PCA) of

\footnotetext{
11 Note that our stellar masses are defined as the integral of the SFR; they are thus about 0.2 dex larger than stellar mass computations which subtract the mass return from stellar evolution to the interstellar medium.
} 


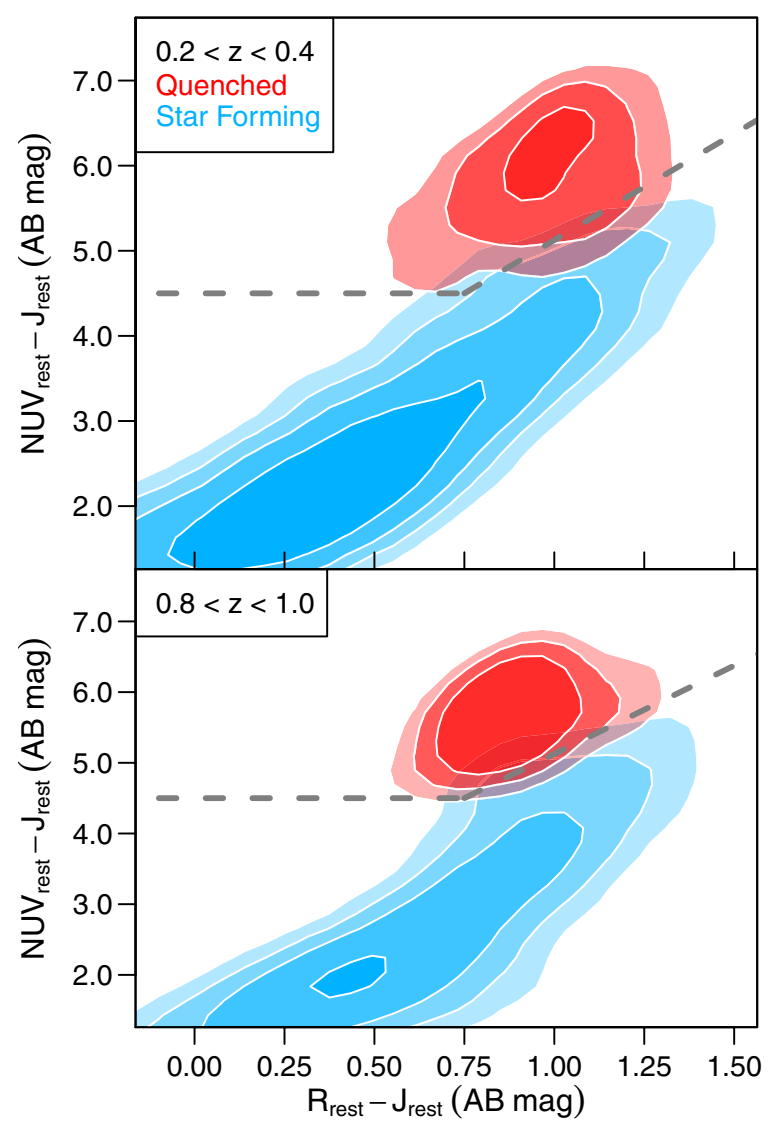

Figure 1. Rest-frame NUV- $J$ vs. $R-J$ color-color distributions of quenched (in red) and star-forming (in blue) galaxies at $0.2<z<0.4$ (top panel) and $0.8<z<1.0$ (bottom panel) in the $I_{814 W}<24$ COSMOS sample. Galaxies are classified as quenched or star-forming according to a threshold in $\mathrm{sSFR}=10^{-11} \mathrm{yr}^{-1}$. This threshold agrees well with the separation in quenched and star-forming that would be derived using the presented color-color diagram. To guide the eyes, we show as a gray dashed line the saddle line between star-forming and quenched samples. Quenched galaxies are largely restricted to $[\mathrm{NUV}-J-2.5 \times(R-J)]>2.6 \mathrm{mag}$ and NUV $-J>4.5 \mathrm{mag}$. The color contours shown are incremented by factors of $2.5,10$, and 25 in number density per bin relative to the baseline, which is a factor of two lower in the high redshift bin.

the previous ZEST version; the morphological classification of the COSMOS sample that we use in this work uses the SVM approach.

Both SVM and PCA method require a training sample to guide the morphological classification. The adopted training set is classified in three main morphological types, i.e., "earlytype" (E/S0) galaxies, "disk" (Sa to Scd) galaxies, and "very late type" ( $\mathrm{Sd} / \mathrm{Irr} / \mathrm{Pec})$ galaxies. The galaxies in the training set were carefully selected as archetypal examples of their classes, well separated from non-class members in (at least) concentration, asymmetry, and Gini coefficient, and spanning a representative range of ellipticities, sizes, and apparent magnitudes (corrected for biases, as discussed in Section 3.2). The morphological classification was performed on the reduced $H S T /$ ACS $I_{814 W}$ frames. Visual inspection of the ZEST + classified sample, and a quantitative inspection based on simulated images, confirms a relatively small $(<15 \%)$ incidence of catastrophic failures in the classification, down to and a much higher frequency of correctly identified morphologies than in our earlier classification attempt using the original version of ZEST (Cameron et al. 2010).

We emphasize that our main findings are not affected by the choice to add a morphological selection to the samples of quenched and star-forming galaxies. We indeed checked that all results stand qualitatively unchanged by removing all morphological constraints, with only minor quantitative differences which do not affect our main conclusions (see also Section 5.3 for a further remark on this point).

\subsection{The Final Galaxy Sample}

The final selection criteria applied to construct the master sample of massive galaxies used in the present analysis were as follows: (1) ZEBRA maximum likelihood photometric redshift in the interval $0.2<z<1.0$ (see Section 2.2); (2) ZEBRA+ maximum likelihood stellar mass greater than $10^{10.5} M_{\odot}$ (see again Section 2.2); (3) ZEST+ morphological type corresponding to E/S0 and Sa-Scd galaxies (see Section 2.3 above); (4) no excessive flux contamination in the ground-based CFHT/Subaru imaging from neighboring objects (as indicated by Capak et al.'s "bad photometry" flag; see Section 2.1); and (5) no contamination by artifacts, cosmic rays, neighboring stars, or from deblending errors in the HST/ACS $I_{814 W}$ imaging (see again Section 2.1). Using these selection criteria, and thanks to the excellent combination of relatively deep ACS images over a $\sim 1.8 \mathrm{deg}^{2}$ field, COSMOS returns a final sample of 11,311 (5355 quenched) galaxies, split in redshifts as follows: 1743 (921) at $0.2<z<0.4,1751(833)$ at $0.4<z<0.6,3093$ (1566) at $0.6<z<0.8$, and 4724 (2035) at $0.8<z<1.0$. The variations of numbers of galaxies in the redshift bins highlight that even COSMOS is not unaffected by cosmic variance. This is a well-known fact for this field, and needs attention in order to perform studies that involve the evolution of the number densities of sources of a given kind. Following the approach that we also adopted in Oesch et al. (2010), we correct for cosmic variance issues as discussed in Section 4.

Finally, we note that, in this paper, we define as "Q-ETGs" galaxies that are quenched, according to our sSFR-based definition of Section 2.2, and have an early-type morphology, according to our classification of Section 2.3.

\section{GALAXY SIZES: BIASES AND CORRECTION FUNCTIONS}

\subsection{Raw Size Measurements}

As indicated above, and as also done in the literature quoted above, we adopt the half-light radius $r_{1 / 2}$ as a measure for the size of galaxies in our sample. While size measurements based on aperture fluxes are well known to prone to systematic biases in the small-size and low surface brightness regimes (e.g., Graham et al. 2005; Cameron \& Driver 2007), we nevertheless favor this technique over a profile-fitting approach (cf. Sargent et al. 2007; Mancini et al. 2010; Cassata et al. 2011; Whitaker et al. 2011) for the present analysis due to its high stability — both the stability of its performance across the full morphological diversity of the high-redshift galaxy population, which becomes increasingly irregular/clumpy and problematic for model fitting codes (Elmegreen et al. 2009; Oesch et al. 2010), and the stability (i.e., predictability) of its systematic biases, which can thus be robustly corrected, as we demonstrate in Section 3.2 below. Conversely, profile-fit-based sizes are themselves prone to increasingly unstable behavior (i.e., large random errors) in the low surface brightness regime (Häussler et al. 2007) and at faint magnitudes; the results of profile fits are unreliable for typical galaxies fainter than $I_{814 \mathrm{~W}} \sim 23 \mathrm{mag}$ in the COSMOS imaging, whereas we want to push our limit 1 mag fainter.

To measure the (aperture) sizes of the galaxies in our sample, we used our custom-built software package ZEST+ (see 
Section 2.3). This also measures galaxy half-light radii within elliptical apertures, providing an alternative to the circular aperture measurements of SExtractor (Bertin \& Arnouts 1996), which are intrinsically limited in their ability to properly characterize elongated systems such as inclined disks. Specifically, given an input measurement of each galaxy's apparent total flux (we employ the $2.5 R_{\text {Kron }}$ value from SExtractor; Kron 1980) and a catalog file highlighting the positions of neighboring objects (again from SExtractor), ZEST+ estimates the local sky background of the galaxy at hand, after replacing the companion galaxies segmentation maps with random sky values, and returns an estimate for the semi-major axis of the corresponding elliptical aperture enclosing half this total flux.

\subsection{Correction Functions for Galaxy Sizes: Dependence on Size, Magnitude, Concentration, and Ellipticity}

It is well established that the brightness of the background sky and the blurring effect of the PSF have the potential to introduce size and surface brightness dependent biases in both the completeness function of galaxy detection and in the recovery of key morphological/structural parameters (cf. Cameron \& Driver 2007; Sargent et al. 2007); hence, the assessment and, if possible, correction of any such biases is an essential requirement for the robust investigation of the evolution of galaxy sizes. Despite this general awareness, studies of galaxy sizes typically limit themselves at quoting global underestimates/overestimates of sizes by a certain fractional value in different regimes of brightness and radial decline of stellar densities (the latter typically represented by Sérsic (1963) indices ranging between $n=1$ and $n>4$, which more or less bracket the surface brightness radial declines of exponential disks and elliptical galaxies at $z=0$ ). Yet the systematic uncertainties may depend also on other parameters, most noticeably the ellipticity and size itself of the stellar structure. Here, we use the approach that we introduced in Cibinel et al. (2012) for the ZENS galaxy sample (Carollo et al. 2012), and apply the appropriate corrections to measured galaxy sizes as a function of galaxy magnitude, size itself, concentration, and ellipticity.

In order to correct for systematic biases in the recovery, from COSMOS ACS $I_{814 W}$ imaging, of galaxy flux and shape parameters, we performed $>2,000,000$ artificial galaxy simulations using GALFIT (Peng et al. 2002). The surface brightness of each model galaxy was defined via an elliptical-isophote, Sérsic profile with intrinsic (input) properties drawn randomly from the following parameter space: $14<I_{814 \mathrm{~W}}<25 \mathrm{mag}$, $0.03<r_{1 / 2}<6$ arcsec, $0<e<0.95$, and $0.2<n<8$. Each model galaxy image was constructed at a pixel scale of 0.03 arcsec, matched to our COSMOS/ACS data, and convolved with a representative ACS $I_{814 W}$ PSF (Rhodes et al. 2007). The model images were then added with Poisson noise to empty regions of sky from the real COSMOS HST/ACS images.

Object extraction was performed on each artificial galaxy using an identical approach to that employed in constructing the COSMOS $I_{814 W}<24$ mag source catalog. That is, we ran SExtractor first in a "bright source mode" (with control parameters: detect_minarea $=140$, detect_thresh $=2.2$, and back_size $=400$ ), and in the event of null detection a second run was initiated in "faint source mode" (with control parameters: detect_minarea $=18$, detect_thresh $=1.0$, and back_size $=100$ ). The relevant photometric and structural parameters output by SExtractor (i.e., the Kron magnitude, Kron half-light radius, and isophotal ellipticity) were recorded for each artificial galaxy thus extracted.

The simulation was then advanced to the ZEST+ analysis stage: as noted earlier, we base our analysis on the elliptical aperture galaxy sizes provided by ZEST+, rather than circular aperture measurements from SExtractor. Hence, as the final stage of our simulation process, each artificial galaxy detected above the sky noise was run through ZEST+ to quantify its concentration index, ellipticity, and half-light radius in a manner consistent with our treatment of the real COSMOS HST/ACS sources.

The suite of artificial galaxy simulations thus compiled allows to establish the impact of observational biases on the measurement of photometric and structural parameters of galaxies in the COSMOS $I_{814 W} H S T /$ ACS catalog by using the output SExtractor and ZEST+ measurements to determine correction functions, for all relevant parameters, at each point of a dense grid in the four-dimensional space of observed magnitude, size, concentration, and ellipticity. Specifically, starting from regularly spaced points on a grid in this four-dimensional observed space, limited by the above boundaries, we selected all models with (output/observed) magnitude within $1 \mathrm{mag}(\Delta \operatorname{mag}=1)$, sizes within $\Delta r=00^{\prime \prime} 015$ (or $\Delta r=0$. 03 ) for effective radii larger than $0^{\prime \prime} .2$ ), ellipticity within $\Delta \epsilon=0.2$, and concentration within $\Delta C=0.5$ of the targeted point on the observed grid. We then computed the median values of the input model parameters that had generated these output (observed) values and derived correction vectors connecting the targeted point on the output grid with the original point on the input grid, i.e., the point with coordinates equal to the median of the input values. A correction vector for each individual galaxy was then obtained by interpolating the correction vectors derived for the grid points.

The resulting correction functions are illustrated in Figure 2, which shows the grid points in the observed radius versus magnitude diagrams, in three bins of concentration (left to right) and ellipticity (top to bottom). The length and direction of the arrows give the point-by-point median correction vectors. The color of the vectors is coded according to the scatter $\Delta_{\text {corr }}$ (in amplitude and/or direction) measured around the median of the correction vector (plotted). The scatter is defined as $\Delta_{\text {corr }}=\sqrt{\left[\sigma\left(r_{1 / 2}\right) /\left\langle r_{1 / 2}\right\rangle\right]^{2}+[\sigma(\mathrm{mag}) /\langle\mathrm{mag}\rangle]^{2}}$, with $\sigma\left(r_{1 / 2}\right)$ and $\left\langle r_{1 / 2}\right\rangle$ and $\sigma$ (mag) and $\langle\mathrm{mag}\rangle$, respectively, being the $1 \sigma$ scatter values and medians of the distributions of recovered halflight radii and magnitudes at the given grid point. The color of the vectors varies from green, for $\Delta_{\text {corr }}<15 \%$, up to red, for $\Delta_{\text {corr }}>50 \%$.

A few well-known trends have been thoroughly commented on in previous studies (e.g., Sargent et al. 2007; Trujillo et al. 2007), e.g., the overestimation of sizes for objects with halflight radii $\leqslant$ FWHM of the PSF, and the underestimation of sizes (and fluxes) for faint, low surface brightness systems with high concentration indices (Mancini et al. 2010). Inspection of the figure reveals that the required correction functions depend on all four parameters (size, magnitude, concentration, and ellipticity). Applying these corrections to the raw measurements is important in order not to introduce systematic biases in the measurements. In this work, we make the step of correcting the observed parameters for each observed galaxy in our COSMOS/ACS catalog, by statistically recovering the intrinsic "true" parameters through interpolation between the vectors in the four-dimensional calibration grid discussed above.

In Figure 3 we show, on the same size-magnitude grid points and fixed concentration/ellipticity panels ("bins"), how 


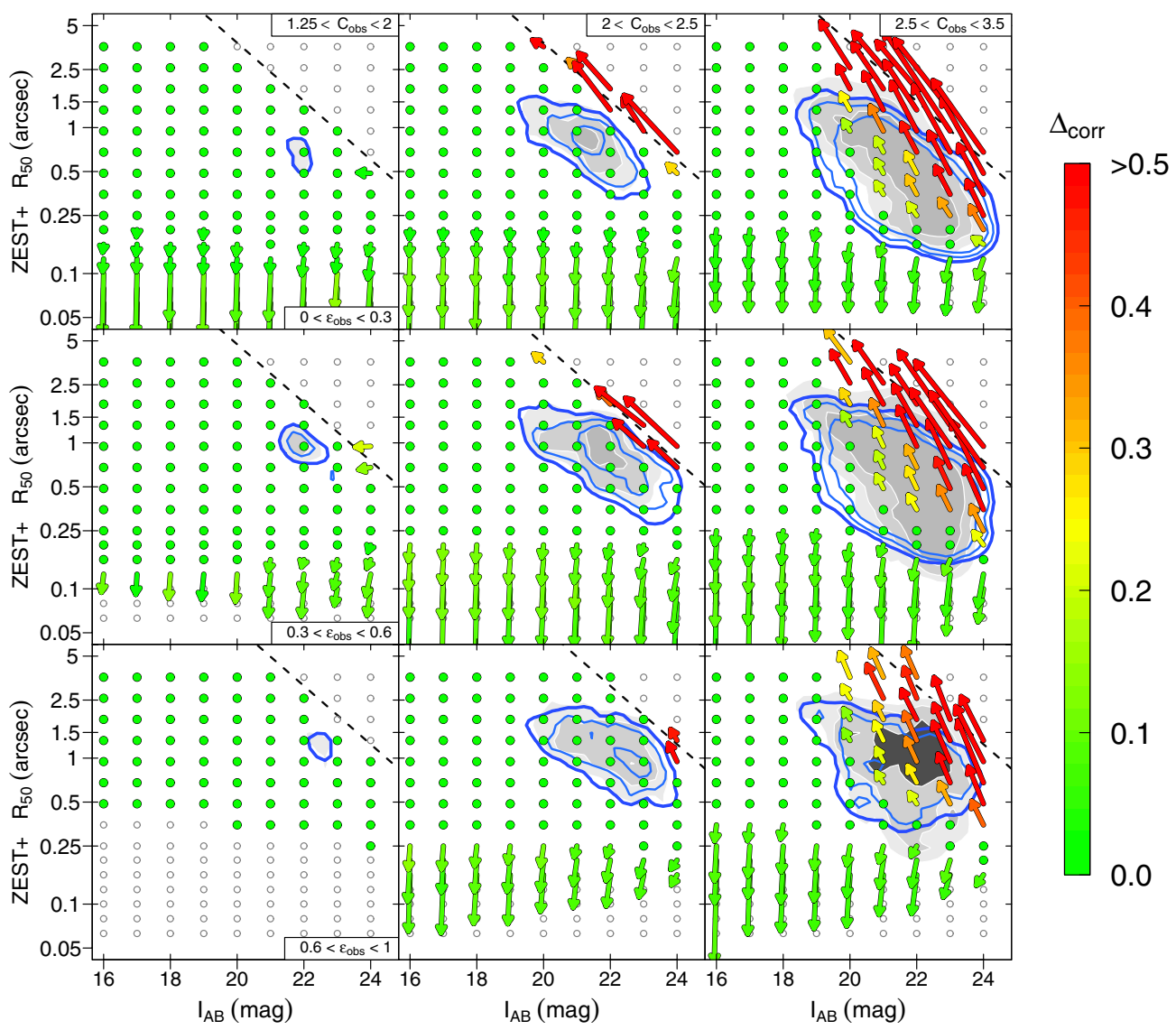

Figure 2. Grid of observed half-light radius and magnitude values, obtained with the aperture approach (ZEST+), showing the correction vectors (represented by the arrows) required at each point of the grid to eliminate systematic biases against these same parameters. The correction vectors specifically give the extent and direction of the average corrections to sizes and magnitudes required at each specific point of the (observed) size-magnitude grid. The nine different panels emphasize the strong influence of profile steepness, and the milder but significant influence of profile ellipticity, in shaping the observational biases. In particular, the correction vectors are shown in three bins of observed concentration index (left to right: $1.25<c_{\mathrm{obs}}<2,2<c_{\mathrm{obs}}<2.5$, and $2.5<c_{\mathrm{obs}}<3.5$ ), and in three bins of observed ellipticity top to bottom: $0<e_{\mathrm{obs}}<0.3,0.3<e_{\mathrm{obs}}<0.6$, and $0.6<e_{\mathrm{obs}}<1.0$ ). Green circles indicate points in the observed size-magnitude grid in which the corrections are $<0.3 \mathrm{mag}$ and $<25 \%$ in radius. The colors of the arrows indicate the degree of uncertainty in the amplitude or direction of the arrows, with a scale from green (negligible errors) to red ( $>50 \%$ error in recovery of the amplitude or direction of the correction). Gray open circles indicate regions of the observed parameter space not populated by enough "observed" artificial galaxies to apply a reliable correction (indicating that galaxies which may populate these grid points would be scattered out, by observational biasses, into other regions of parameter space; see also Figure 3 and related discussion in the text). The blue contour lines and the gray-shaded contours in each panel show respectively the distributions of galaxies in our final $0.2<z<1.0$ COSMOS sample for uncorrected and corrected measurements (in steps of a factor of two in number density per contour). The dashed line in the top right corner of the panels shows the surface brightness limit for the $I_{814 W}<24$ COSMOS sample.

systematic measurement errors in concentration and ellipticity scatter model galaxies out of their input/true bins and into incorrect bins of these parameters. The main point of the figure is to give a "glimpse impression" of problematic (yellow to red) regions in the four-dimensional parameter space of magnitude, size, concentration, and ellipticity. In detail, filled green circles in the panels indicate grid points in which model galaxies simulated with these parameters are more than $50 \%$ of the times correctly recovered within the original bin of concentration and ellipticity, and with a negligible scatter $(\Delta C<0.3$ and $\Delta \epsilon<0.2)$ relative to the true input parameters. Filled red circles indicate grid points in which a similarly large fraction of model galaxies simulated with these parameters is recovered within the original broad bin of concentration and ellipticity, but with a large scatter in concentration index $(\Delta C>0.3)$ and in ellipticity $(\Delta \epsilon>0.2)$ relative to the true input parameters. Squares identify grid points, in a given bin of observed ellipticity and concentration, that are dominated by a fraction of at least $50 \%$ (yellow) to $>90 \%$ (red) of model galaxies which originate in a different bin of concentration or ellipticity, and are scattered into the observed grid point by measurement errors. ${ }^{12}$ Finally, arrows indicate grid points from which galaxies that are born with those given ellipticity/concentration parameters "disappear" from that grid point, and are observed with substantially different ellipticity and/or concentration parameters. Specifically, solid arrows indicate points of the size-magnitude grid in a given input/true bin of ellipticity and concentration, from which at least $50 \%$ of model galaxies born in this bin are scattered out from it, and end up, due to systematic measurement errors, being "observed" in other (incorrect) bins of ellipticity and concentration. Colors from yellow to red indicate an increasing fraction, above the threshold of 50\% required to plot an arrow (yellow), of outscattered model galaxies (with red showing a loss of $>90 \%$ of galaxies from the grid point). Empty arrows indicate grid points from which similar fractions of galaxies ( $>50 \%$, yellow, up to $>90 \%$,

\footnotetext{
12 Note that red/green circles can be surrounded by squares, indicating that galaxies born with the given grid point parameters are well recovered within the same ellipticity/concentration bin, but, to this indigenous population, a population of interloper galaxies is added, due to scattering of galaxies into the given grid points from other ellipticity/concentration bins.
} 


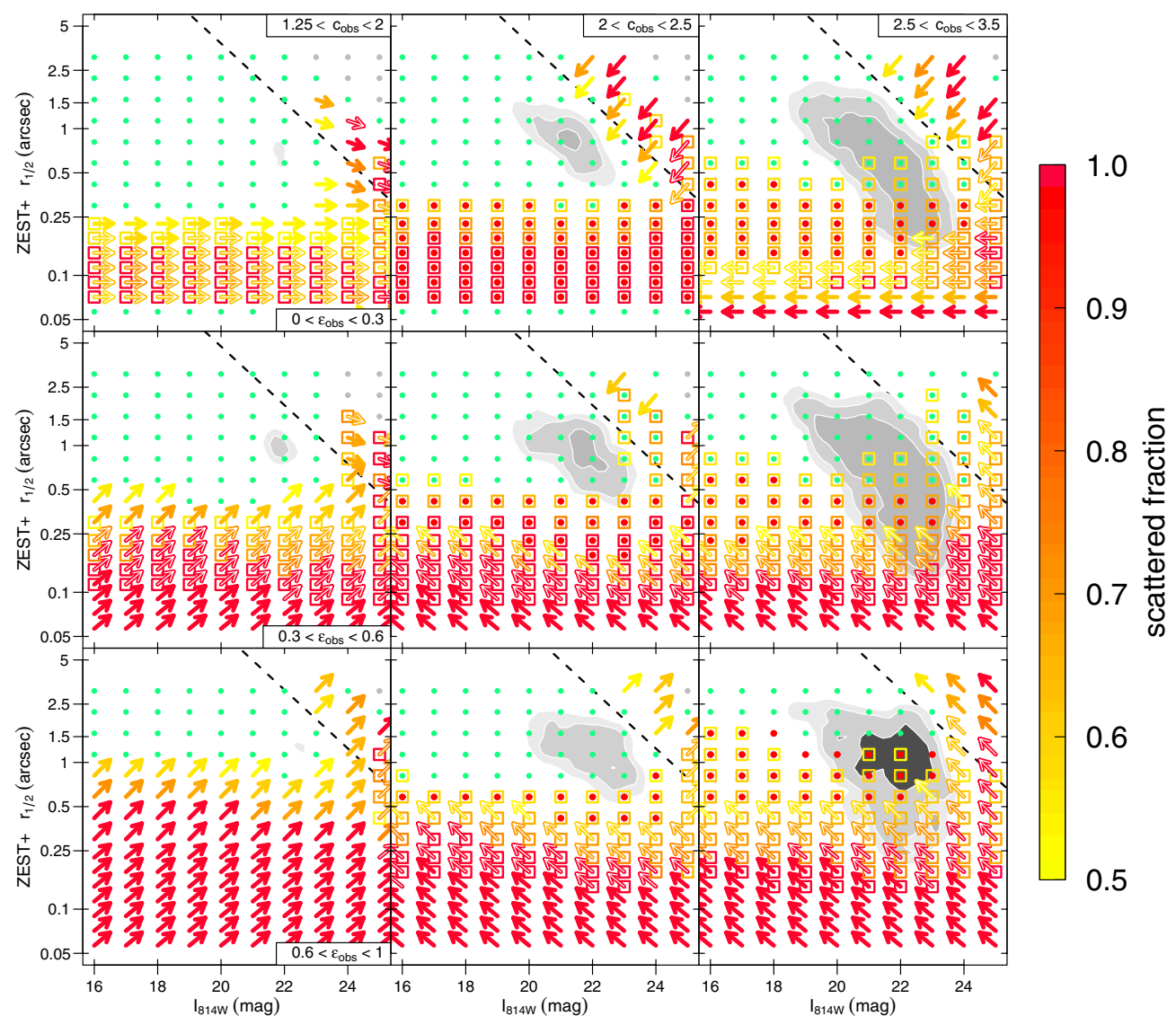

Figure 3. Grid of true/input half-light radius and magnitude values, obtained with the aperture approach (ZEST+), shown as in Figure 2 split into three bins of observed concentration index (left to right: $1.25<c_{\mathrm{obs}}<2,2<c_{\mathrm{obs}}<2.5$, and $2.5<c_{\mathrm{obs}}<3.5$ ), and into three bins of observed ellipticity (top to bottom: $0<e_{\mathrm{obs}}<0.3$, $0.3<e_{\mathrm{obs}}<0.6$, and $0.6<e_{\mathrm{obs}}<1.0$ ). Filled green circles show grid points in which more than $50 \%$ of model galaxies born with these given input concentration $C$ and ellipticity $\epsilon$ parameters, are correctly recovered with a negligible scatter relative to these input parameters (namely, $\Delta C<0.3$ and $\Delta \epsilon<0.2$ ). Filled red circles show grid points in which $>50 \%$ of model galaxies simulated with these parameters are recovered within the original broad bin of concentration and ellipticity (i.e., same panel), but with a large scatter in concentration index $(\Delta C>0.3)$ and in ellipticity $(\Delta \epsilon>0.2)$ relative to the true input parameters. Squares show grid points in which $>50 \%$ (yellow, up to $>90 \%$, red) of model galaxies are detected at that grid point but originate from a different bin (panel) of concentration or ellipticity. Solid arrows show grid points from which at least $50 \%$ (yellow, up to $>90 \%$, red) of model galaxies born there are scattered out from the given ellipticity/concentration bin (i.e., panel) and are "observed" in a different panel of ellipticity and concentration. Empty arrows show grid points from which similarly color-code fractions of galaxies which are born with those given ellipticity/concentration values remain within the same ellipticity/concentration panel bin, but are recovered with errors in concentration $\Delta C>0.3$ and ellipticity $\Delta \epsilon>0.2$, respectively. The direction of the arrows visualizes the global "scattering direction," due to measurement errors, of galaxy models out of that bin. In particular, left/rightward oriented horizontal arrows indicate recovery errors in concentration only, and specifically scattering toward lower/higher concentrations, respectively. The $45^{\circ}$ inclined left/rightward and up/downward directed arrows indicate recovery errors in both concentration and ellipticity, and precisely toward lower/higher concentrations and lower/higher ellipticities, respectively. Remaining symbols in the figure are as in Figure 2.

red) which are born with those given ellipticity/concentration values remain within the same ellipticity/concentration bin, but are recovered with errors in concentration $\Delta C>0.3$ and ellipticity $\Delta \epsilon>0.2$, respectively. As in Figure 2, the gray contours show the corrected $0.2<z<1.0, I_{814 W}<24$ mag COSMOS galaxy population (with a change in number density of a factor of two between density contours). We discuss in Appendix B some consistency checks that ensure that the derived correction functions return quantities, including sizes, that are free from further biases.

We emphasize that galaxies in some regimes of parameter space cannot be recovered, and thus some systematic biases cannot be corrected. First, at very low surface brightnesses, the original Kron flux underestimation causes objects to fall entirely below our $I_{814 \mathrm{~W}}<24 \mathrm{mag}$ catalog selection limit. These regions of classical low surface brightness incompleteness are identifiable in Figures 2 and 3. This incompleteness effect has a minimal impact on the observed magnitude-size distributions in COSMOS, hampering the observability of only the very largest galaxies with magnitudes fainter than $I_{814 W} \sim 23.5 \mathrm{mag}$, which motivates our initial sample selection criteria. Given its nature, this residual bias has no impact on the main results that we highlight in this paper. Second, at very small sizes, due to PSF broadening, galaxies are scattered in regions of low concentration and low ellipticity values from bins of high concentration and ellipticity; there is thus an intrinsic degeneracy between small galaxies which are intrinsically diffuse and round, and galaxies which appear so, although they are in reality more concentrated and elongated than they appear. These residual biases must be kept in mind when analyzing galaxy samples; again, they do not affect however the results that we present in this paper.

Finally, in Appendix $\mathrm{C}$ we show that corrections to galactic sizes as a function of magnitude, size itself, concentration, and ellipticity are needed not only for aperture size measurements, but generally also for sizes that are derived from analytical fits to the galaxy surface brightness distributions. As a showcase we present in this Appendix the correction functions, in a similar way as in Figures 2 and 3, but for sizes obtained using the public code GALFIT (Peng et al. 2002). The GALFIT analysis 

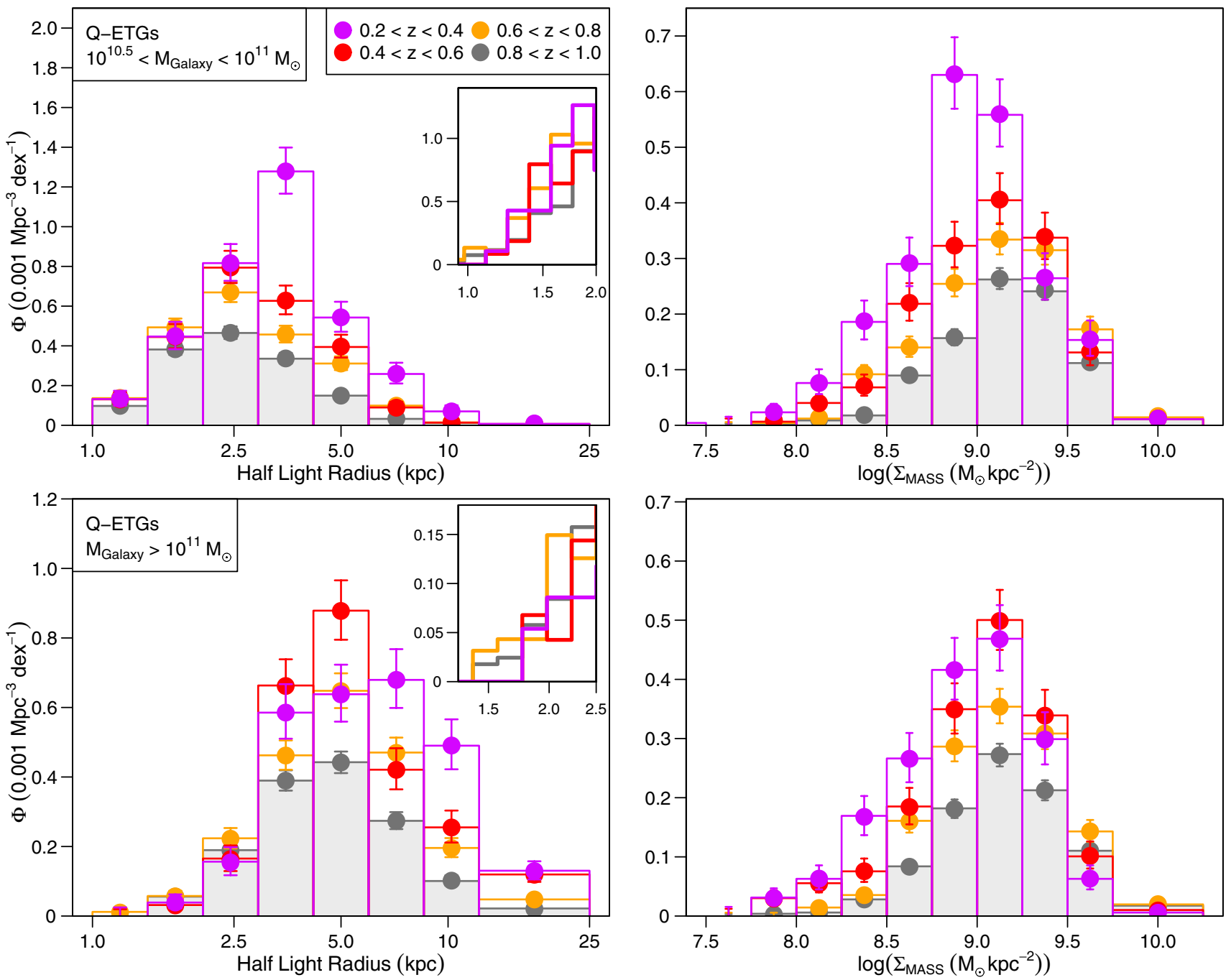

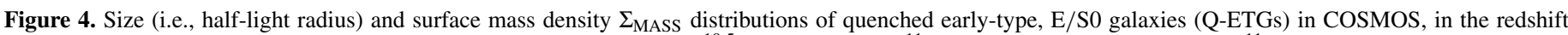

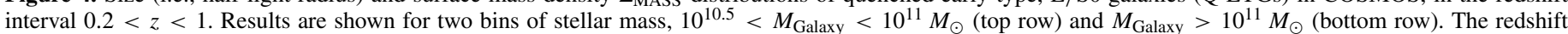

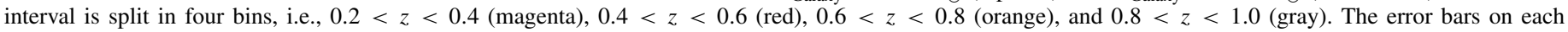

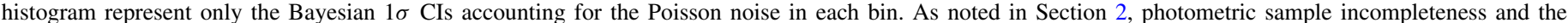

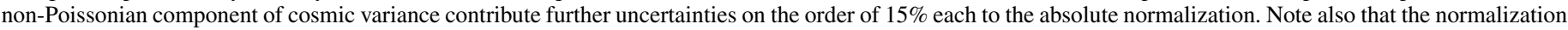

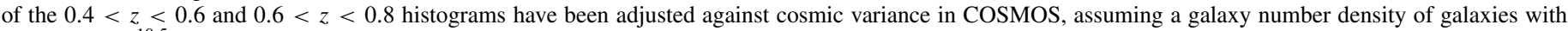

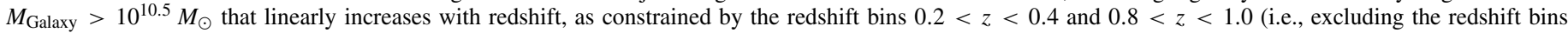

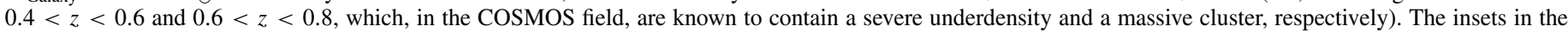

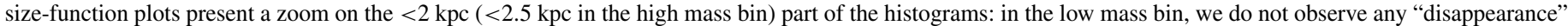

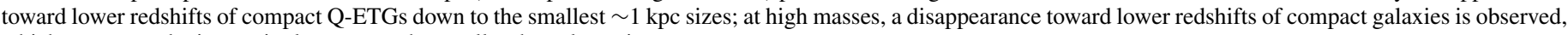
which appears to be increasingly stronger the smaller the galaxy size.

in the Appendix uses the same set of simulated galaxies which were used to correct the ZEST+ size measurements that we use in our analysis. We note that the trends and strengths of the correction vectors for the GALFIT sizes differ from those reported in this section for the ZEST+ sizes: GALFIT does better than ZEST+ in the regime of small sizes (i.e., smaller than the PSF), but is less accurate in recovering sizes of large, low surface brightness galaxies. Our main message here is twofold: first, in order to study galaxy populations spanning a large range of sizes and surface brightness, both aperture-based and the modelfit size measurements require corrections. Second, once these corrections are applied, galaxy sizes derived with either of the two approaches are robust and in very good agreement with each other, as shown in Appendix C.

We therefore stress that the results that we present in this paper do not depend on our specific choice for how to quantify galaxy sizes. We also expect our results to be unaffected by "morphological $K$-corrections," as remarked already in Section 1, due to the lack of strong color gradients in high- $z$ Q-ETG demonstrated in several earlier works (e.g., Toft et al. 2007; Guo et al. 2011).

\section{EVOLUTION IN THE NUMBER DENSITIES OF Q-ETGs AT FIXED MASS, SIZE, AND SURFACE MASS DENSITY}

Figure 4 shows the number density of Q-ETGs in COSMOS at fixed size (i.e., the size function $\Phi_{r_{1 / 2}}$; left-hand panels) and surface mass density $\Sigma_{\text {MASS }}$ (i.e., the $\Sigma_{\text {MASS }}$ function $\Phi_{\Sigma_{\text {MASS }}}$; right-hand panels) in four bins of redshift: $0.2<z<0.4$ (magenta), $0.4<z<0.6$ (red), $0.6<z<0.8$ (orange), and $0.8<z<1.0$ (gray).

We restrict our investigation to two fixed intervals of stellar mass above the effective completeness limit at $z=1$ for the spectro-morphological "early-type galaxy" class in the $I_{814 W}<$ 

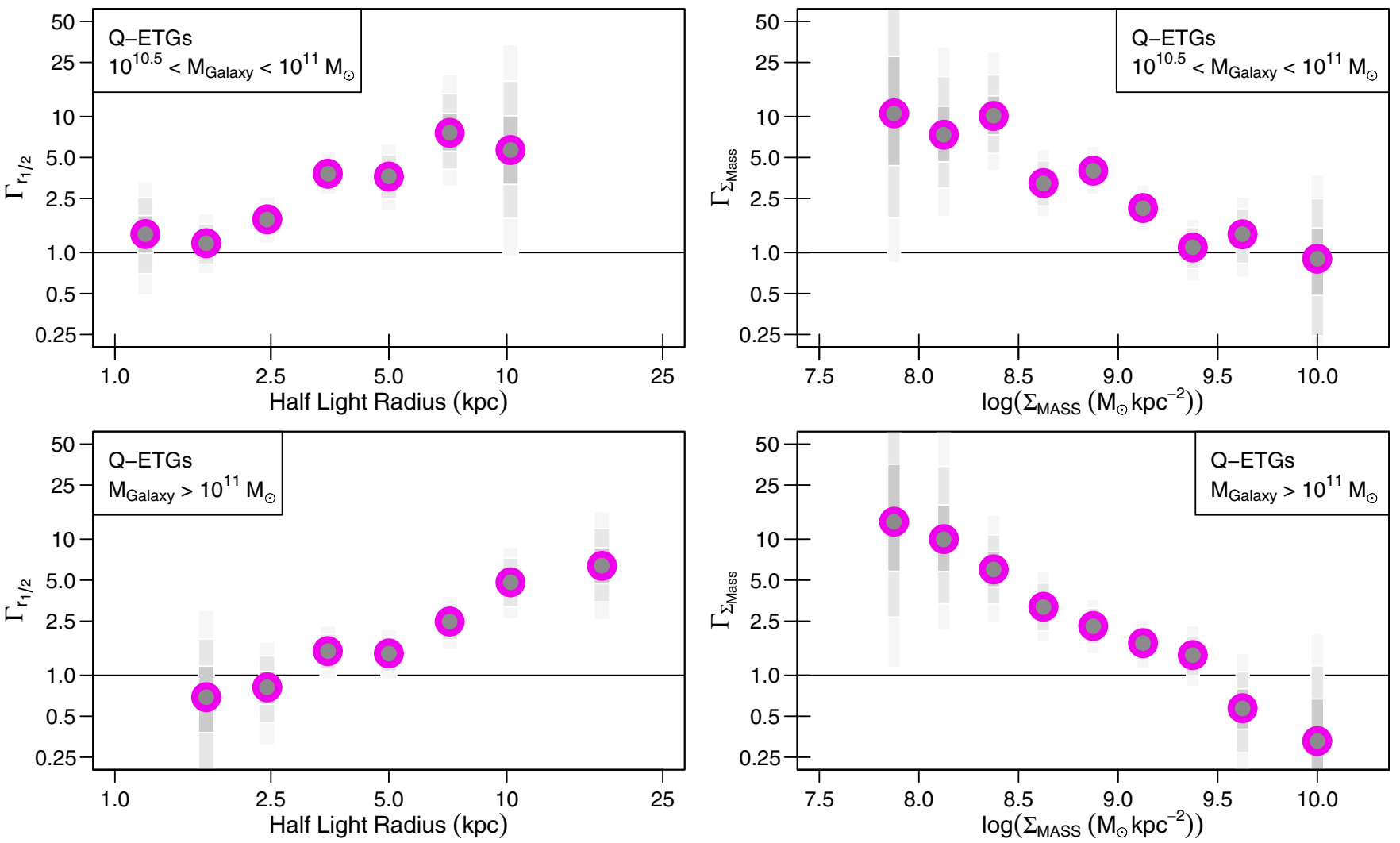

Figure 5. Growth factors between $z=1$ and $z=0.2, \Gamma_{r_{1 / 2}}$ and $\Gamma_{\Sigma_{\mathrm{MASS}}}$, of the number density of quenched early-type galaxies (Q-ETGs) at fixed size (left) and $\Sigma_{\mathrm{MASS}}$ (right). Results are shown separately for the low mass bin, $10^{10.5}<M_{\text {Galaxy }}<10^{11} M_{\odot}$ (top row) and for the high mass bin, $M_{\text {Galaxy }}>10^{11} M_{\odot}$ (bottom row). The

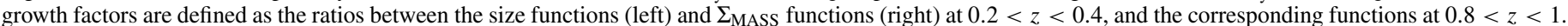
The dark-, intermediate-, and light-gray vertical bars trace the $1 \sigma, 2 \sigma$, and $3 \sigma$ Bayesian confidence intervals on these factors, respectively, with the medians marked by the magenta+gray data points. The coloring of these data points is used as a reminder that they express the ratios between the magenta and gray curves in Figure 4 .

24 COSMOS catalog (see Oesch et al. 2010; Ilbert et al. 2010). In particular, the large size of the COSMOS sample enables us to split the total sample of massive, $>10^{10.5} M_{\odot}$ Q-ETGs at these redshifts into two distinct mass bins straddling across the $M_{\text {Galaxy }}=10^{11} M_{\odot} \sim M^{*}$ value. We thus define a "low mass bin," with $10^{10.5}<M_{\text {Galaxy }}<10^{11} M_{\odot}$ (top row in Figure 4), and a "high mass bin," with $M_{\text {Galaxy }}>10^{11} M_{\odot}$ (bottom row). We have chosen a linear scale on the $y$-axis to display the size and $\Sigma_{\text {MASS }}$ functions $\Phi$; the error bars, however, reflect the Bayesian $1 \sigma$ confidence intervals (CIs) on the number density in each bin treated as a Poisson rate parameter (obtained from inversion of the posterior probability distribution for $\Phi$, given the observed number of objects in that bin and the improper uniform prior; see Kraft et al. 1991 for details).

Note that, in order to correct for cosmic variance effects in the COSMOS field, well known to contain a severe underdensity and a massive cluster at $z \sim 0.5$ and $z \sim 0.75$, respectively (see, e.g., Scarlata et al. 2007b; Oesch et al. 2010), we have followed the approach of Oesch et al. (2010) and normalized the total number density $\Phi\left(M_{\text {Galaxy }}>10^{10.5} M_{\odot}\right)$ of galaxies of all morphological types in the $0.4<z<0.6$ and $0.6<z<0.8$ redshift bins, and with stellar masses $M_{\text {Galaxy }}>10^{10.5} M_{\odot}$, against a linear interpolation with redshift of the number densities of corresponding galaxies in the $0.2<z<0.4$ and $0.8<z<1.0$ redshift bins.

To better highlight the global evolution since $z=1$ of the size and $\Sigma_{\text {MASS }}$ functions, we plot in Figure 5 the ratios between these functions in the lowest $(0.2<z<0.4)$ and highest $(0.8<z<1)$ redshift bins of our analysis. These ratios defined respectively as

$$
\Gamma_{r_{1 / 2}}=\frac{\Phi_{r_{1 / 2}}(0.2<z<0.4)}{\Phi_{r_{1 / 2}}(0.8<z<1)}
$$

and

$$
\Gamma_{\Sigma_{\mathrm{MASS}}}=\frac{\Phi_{\Sigma_{\mathrm{MASS}}}(0.2<z<0.4)}{\Phi_{\Sigma_{\mathrm{MASS}}}(0.8<z<1)}
$$

highlight in a straightforward manner the rate of growth in the number densities of Q-ETGs in each bin of size and $\Sigma_{\text {MAss }}$.

At the highest stellar masses, i.e., in the $M_{\text {Galaxy }}>10^{11} M_{\odot}$ bin of our analysis, we detect a decrease from $z=1$ to $z=0.2$ of about $30 \%-40 \%$ of the smallest and densest Q-ETGs, i.e., of high-mass Q-ETGs with half-light radii smaller than $\sim 2 \mathrm{kpc}$. Note that in our data this is only seen at the $1 \sigma$ level. Nevertheless, this result, obtained within a self-consistent data set, formally agrees with the several works which report a decreasing number density of compact galaxies with increasing age of the universe (see references above). At these high masses, the disappearance toward lower redshifts of compact galaxies seems to be increasingly larger the smaller the galaxy size, as shown in the inset in the relevant size-function plot of Figure 4, which zooms on the $<2.5 \mathrm{kpc}$ scales.

The analysis of our low mass bin at $10^{10.5}<M_{\text {Galaxy }}<$ $10^{11} M_{\odot}$, i.e., just below $M^{*}$, also shows surprises. The number density of the $r_{1 / 2} \leqslant 2 \mathrm{kpc}$ Q-ETGs remains remarkably stable throughout the $z=1 \rightarrow 0.2$ redshift range. At these masses, there is essentially no change in the number density of the most compact Q-ETGs since $z \sim 1$ down to $z \sim 0.2$, and thus no 

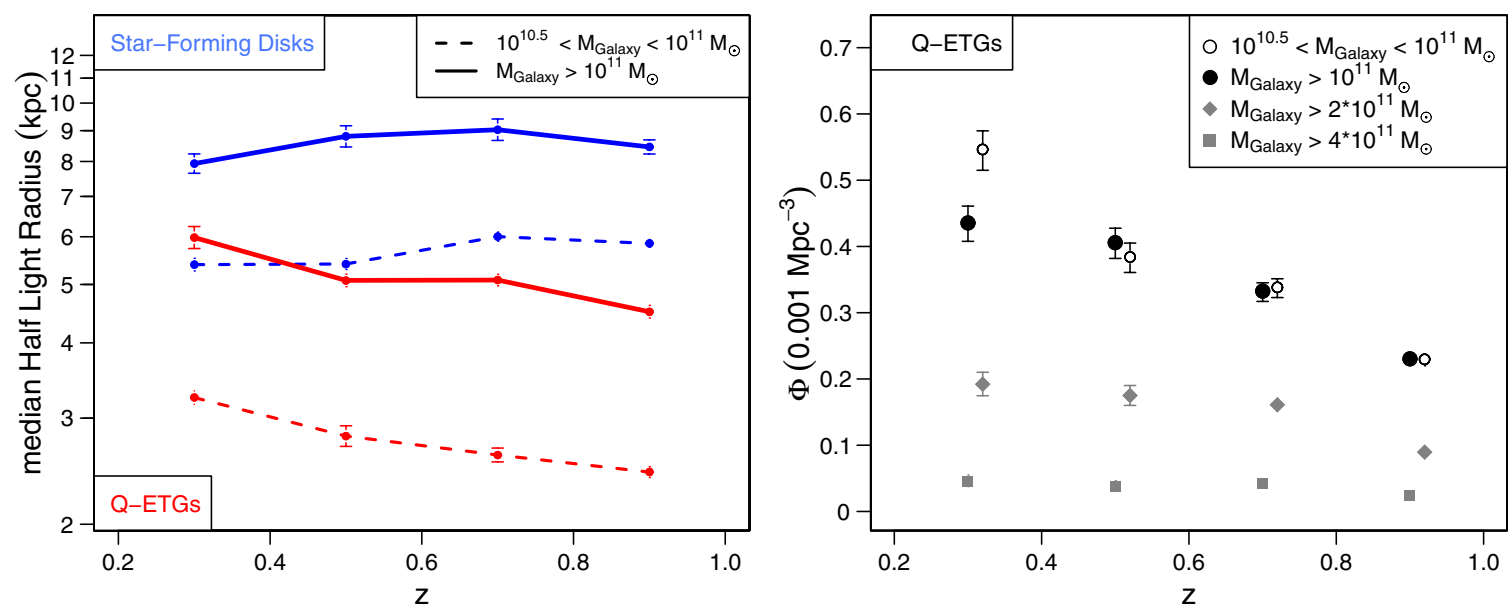

Figure 6. Left panel: the median size (half-light radius) of Q-ETGs (in red) and star-forming disks (in blue), as a function of redshift, for the high mass bin $\left(M_{\text {Galaxy }}>10^{11} M_{\odot}\right.$; solid lines) and low mass bin $\left(10^{10.5} M_{\odot}<M<10^{11} M_{\odot} ;\right.$ dashed lines). Right panel: the number density of Q-ETGs as a function of redshift in the low (empty circles) and high mass (solid circles) bin. Error bars indicate $1 \sigma$ uncertainties on the median values. We also plot the number density evolution of $2 \times 10^{11} M_{\odot}$ (diamonds) and $4 \times 10^{11} M_{\odot}$ (squares) to show that most of the growth in our high mass bin occurs peaked at $\sim 10^{11} M_{\odot} \sim M^{*}$.

evidence for any disappearance of compact galaxies due to an increase in their individual sizes across this time span. This result is not affected by our binning in size: it holds down to the smallest $\sim 1 \mathrm{kpc}$ sizes, as shown in the inset in the relevant size-function panel of Figure 4, which plots again a zoom-in of the small-scale size function (this time in the $<2 \mathrm{kpc}$ regime). Note that we ascertain this constancy self-consistently within the COSMOS sample, without recurring to comparisons between different studies or data sets. This ensures no spurious effects introduced by different approaches for computing the sizes, and by different PSFs, noise properties, systematic effects, different depths, and other such potential complications.

Similarly striking in Figure 5 is that, in both mass bins, we do observe a strong evolution in the size and $\Sigma_{\text {MASS }}$ functions of Q-ETGs, with a marked dependence on galaxy size in both mass bins for the population growth rates $\Gamma_{r_{1 / 2}}$. This evolution involves however not the small-size, compact Q-ETG population, but rather the number densities of large-size (and thus low $\Sigma_{\mathrm{MASS}}$ ) Q-ETGs. In particular, the inspection of Figure 5 shows that, in both low and high mass bins, a substantial increase in number density of large-size Q-ETGs has taken place since $z=1$ : the growth rates $\Gamma_{r_{1 / 2}}$ rise, respectively, in the low mass and high mass bins, from of order unity (i.e., the null growth) and from the negative rate of $\sim 0.3$ at $r_{1 / 2} \lesssim 2 \mathrm{kpc}$, as reported above, up to $\sim 5-6$ by $r_{1 / 2} \gtrsim 5 \mathrm{kpc}$ (low mass bin) and $r_{1 / 2} \sim 12 \mathrm{kpc}$ (high mass bin). This corresponds to similar growth factors from $z=1$ to $z=0.2$ for the population of $l o w-\Sigma_{\text {MASS }}$ galaxies, i.e., growth rates $\Gamma_{\Sigma_{\mathrm{MASS}}} \sim 5$ from $z=1$ to $z=0.2$ of galaxies with $\Sigma_{\text {MASS }} \leqslant 9 M_{\odot} \mathrm{kpc}^{-2}$.

The significant increase in number density of large-size Q-ETGs with cosmic time between $z \sim 1$ and $z \sim 0.2$ implies that the newly quenched, large $Q$-ETGs (hereafter NQ-ETGs ${ }^{13}$ ) lead to a substantial increase in the median half-light size for the whole Q-ETG population. The proportional increase in median size is comparable in the high mass and low mass bins, and equal to a factor of $\sim 1.3$ over the redshift span of our analysis.

13 Note that the acronym "NQ-ETGs" is used to indicate ETGs that are newly quenched at any given epoch, not an observational galaxy sample selected to span a specific range of values in some measured parameters. In Figure 8, the population of NQ-ETGs which emerges between our highest and lowest redshift bin is shown, in each of the mass bins, by the black hatched histogram that results from subtracting the $0.8<z<1$ size function from the $0.2<z<0.4$ size function.
Specifically, as shown in Figure 6 (left panel), the median size of Q-ETGs grows from $4.5 \pm 0.1 \mathrm{kpc}$ to $6.0 \pm 0.3 \mathrm{kpc}$ in the high mass bin (solid red line), and from $2.4 \pm 0.1 \mathrm{kpc}$ to $3.2 \pm 0.1 \mathrm{kpc}$ in the low mass bin (dashed red line), respectively; these imply a formal growth as approximately $(1+z)^{-0.70 \pm 0.15}$. Correspondingly, and consistently with other analyses, there is an increase by a factor of $\sim 2-2.5$, between $z \sim 1$ and $z \sim 0.2$, in the overall number density of $M_{\text {Galaxy }}>10^{10.5} M_{\odot}$ Q-ETGs. This is shown in the right panel of Figure 6, where we plot the number density of Q-ETGs, summed over all sizes, as a function of redshift; the result is shown separately for our low mass $\left(10^{10.5} M_{\odot}<M_{\text {Galaxy }}<10^{11} M_{\odot}\right.$; empty points $)$ and high mass $\left(M_{\text {Galaxy }}>10^{11} M_{\odot}\right.$; solid points) bins. As discussed in Section 1, these number density growth factors for Q-ETGs agree well with the measured evolution of the mass function of quiescent galaxies since $z \sim 1$.

Note that in Figure 6 the increase since $z=1$ of the number density of Q-ETGs with masses integrated above $10^{11} M_{\odot}$ contrasts the well-known constancy in number density of the most massive Q-ETGs, with masses of the order of 2-4× $10^{11} M_{\odot}$ (see also Cimatti et al. 2006; Scarlata et al. 2007b; Pozzetti et al. 2010; Ilbert et al. 2013). This highlights that the number growth from $z=1$ to $z=0$ of the Q-ETG population at galaxy masses above $10^{11} M_{\odot}$ occurs predominantly within a very narrow mass peak around $\sim 10^{11} M_{\odot} \sim M^{*}$. This reinforces the notion that the probability for a galaxy to survive quenching and keep living as a star-forming galaxy drops exponentially with increasing stellar mass and becomes negligible above $M^{*}$ (see Peng et al. 2010).

Keeping in mind that we observe little or no decrease in the number densities of the smallest and most compact Q-ETGs since $z=1$, the key question to answer is what is the main cause for the appearance of large Q-ETGs at later times-appearance which is largely responsible for the measured increase in the median size of the whole population of such quenched systems with an early-type morphology, whereas the growth of individual galaxies has apparently no effect on it.

\section{DISCUSSION: THE EMERGENCE OF LARGE Q-ETGs AT LATE EPOCHS}

We can summarize our findings above with two simple statements: (1) the number density of compact $<2 \mathrm{kpc}$ Q-ETG 
Table 1

Median Rest-frame $(U-V)$ Colors of Compact (Low Mass Bin: $<2 \mathrm{kpc}$; High Mass Bin: <2.5 kpc) and Large (>4 kpc) Q-ETGs

\begin{tabular}{lccccc}
\hline \hline Redshift & \multicolumn{2}{c}{$10^{10.5}<M / M_{\odot}<10^{11}$} & & \multicolumn{2}{c}{$M / M_{\odot}>10^{11}$} \\
\cline { 2 - 3 } \cline { 5 - 6 } & $r_{1 / 2}<2 \mathrm{kpc}$ & $r_{1 / 2}>4 \mathrm{kpc}$ & & $r_{1 / 2}<2.5 \mathrm{kpc}$ & $r_{1 / 2}>4 \mathrm{kpc}$ \\
\hline $0.2 \leqslant z<0.4$ & $1.57 \pm 0.01$ & $1.49 \pm 0.01$ & & $1.56 \pm 0.04$ & $1.55 \pm 0.01$ \\
$0.4 \leqslant z<0.6$ & $1.49 \pm 0.01$ & $1.45 \pm 0.01$ & & $1.52 \pm 0.04$ & $1.48 \pm 0.01$ \\
$0.6 \leqslant z<0.8$ & $1.44 \pm 0.01$ & $1.41 \pm 0.01$ & & $1.53 \pm 0.02$ & $1.53 \pm 0.01$ \\
$0.8 \leqslant z<1.0$ & $1.42 \pm 0.01$ & $1.41 \pm 0.01$ & & $1.42 \pm 0.02$ & $1.50 \pm 0.01$ \\
\hline
\end{tabular}

remains stable since $z=1$, with at most a very modest decrease with decreasing redshift of the order of $30 \%$ for the most massive $>10^{11} M_{\odot}$ Q-ETGs; and (2) most of the growth of the median size of the $>10^{10.5} M_{\odot}$ Q-ETG population is due to the appearance at later times of large(r) Q-ETGs.

On the basis of the current evidence, it is possible of course that the Q-ETG population grows in size with cosmic time in a coordinated manner, so as to keep constant the number density of the smallest/densest galaxies, which should be created at the same rate at which they would be shifted to the higher size bins. While it seems somewhat contrived that such conspiracy may take place, we note that in this case the most compact galaxies would be the last that have been quenched, and thus they should be systematically younger than average. Current analyses of the stellar population ages in the $z \sim 2$ (Saracco et al. 2011) and $z=0$ (van der Wel et al. 2009) Q-ETG populations find hints for an effect in the opposite direction, i.e., the most compact galaxies look older, not younger, than their larger counterparts.

\subsection{The Rest-frame $(U-V)$ Colors of Compact and Large Q-ETGs}

We search for possible trends in stellar population ages of compact and large Q-ETGs in our own data: in Figure 7 (top), we plot the distributions of the rest-frame $(U-V)$ colors of the low (two leftmost columns) and high (two rightmost columns) mass bins, split into two subsamples with sizes, respectively, $<2 \mathrm{kpc}$ (for the low-mass galaxies, and $<2.5 \mathrm{kpc}$, for the high-mass galaxies) and $>4 \mathrm{kpc}$. The median colors of both populations in each redshift bin are listed in Table 1.

It is immediately evident that there is a trend in the same direction as the previous studies at lower and higher redshifts. The colors of the most compact Q-ETGs become on average increasingly redder toward lower redshifts. Specifically, the color difference between compact Q-ETGs at $z=1$ and at $z=0.2$ is $\Delta(U-V) \sim 0.16$, which is in very good agreement with the expected color change for a single stellar population that formed at $z \sim 2$ and passively evolves between these two epochs. Furthermore, at low masses, compact Q-ETGs are systematically redder than their large counterparts at similar masses, strengthening the interpretation that the former are older than the latter (see also Shankar \& Bernardi 2009; Saracco et al. 2011).

We test that variations in stellar mass within the formal mass bins do not impact the conclusions above concerning the relative average ages for the compact and large Q-ETG populations. Also in Figure 7 (bottom) we show the distributions of stellar masses, within each redshift bin and formal bin of stellar mass, for the same compact and large samples of Q-ETGs of the color analysis. The median stellar masses of these distributions are listed in Table 2. In each formal bin of mass, the median stellar mass of the compact population is very constant with redshift. There is understandably a trend for the compact
Table 2

Median Stellar Masses within the Corresponding Mass Bins for the Samples of Table 1 of Compact and Large Q-ETGs

\begin{tabular}{lccccc}
\hline \hline Redshift & \multicolumn{2}{c}{$10^{10.5}<M / M_{\odot}<10^{11}$} & & \multicolumn{2}{c}{$M / M_{\odot}>10^{11}$} \\
\cline { 2 - 3 } \cline { 5 - 6 } & $r_{1 / 2}<2 \mathrm{kpc}$ & $r_{1 / 2}>4 \mathrm{kpc}$ & & $r_{1 / 2}<2.5 \mathrm{kpc}$ & $r_{1 / 2}>4 \mathrm{kpc}$ \\
\hline $0.2 \leqslant z<0.4$ & $10.69 \pm 0.03$ & $10.83 \pm 0.02$ & & $11.06 \pm 0.04$ & $11.34 \pm 0.02$ \\
$0.4 \leqslant z<0.6$ & $10.71 \pm 0.02$ & $10.82 \pm 0.02$ & & $11.11 \pm 0.04$ & $11.32 \pm 0.02$ \\
$0.6 \leqslant z<0.8$ & $10.69 \pm 0.01$ & $10.83 \pm 0.03$ & & $11.07 \pm 0.02$ & $11.36 \pm 0.02$ \\
$0.8 \leqslant z<1.0$ & $10.70 \pm 0.01$ & $10.89 \pm 0.02$ & & $11.07 \pm 0.01$ & $11.32 \pm 0.01$
\end{tabular}

Q-ETG sample to have, within each formal mass bin, a slightly smaller median mass than the large Q-ETG population. This goes, however, in the direction of decreasing, if anything, the color difference between old compact versus young large Q-ETGs. We thus conclude that the color difference that we have detected between compact and large Q-ETGs in the low mass bin is a genuine stellar population effect, indeed consistent with a younger average age of large relative to compact Q-ETGs. In the high mass bin the two populations, compact and large, have much more similar colors. At these high masses, mergers play a much larger role. We will further comment on this below.

In light of these results, a more plausible interpretation is that a static rather than dynamic equilibrium holds for the number density of $<2 \mathrm{kpc}$ Q-ETGs (i.e., the population of compact QETGs remains virtually unchanged between $z=1$ and $z=0.2$, without either creation of new compact Q-ETGs or growth of their individual sizes over this time period). The emergence at low redshifts of the population of large and diffuse Q-ETGs, seen in both our low and high mass bins, therefore results from larger star-forming galaxies experiencing the quenching trauma at later times.

\subsection{Comparison of Typical Sizes of Newly Quenched Galaxies at Different Redshifts}

We can use the size functions of Q-ETGs of Figure 4 to derive, in both our mass bins, empirical estimates for the size functions of the newly quenched populations over the $z=1 \rightarrow 0.2$ time period, and their median sizes at the median redshift of $z \sim 0.6$. These estimates are readily defined through the difference between the $0.2<z<0.4$ and the $0.8<z<1.0$ size functions of Q-ETGs of Figure 4, replotted in Figure 8 as solid magenta and gray histograms, respectively (left and right panels refer respectively to the low and high mass bins). The size functions of the NQ-ETGs are shown as black hatched histograms; the corresponding median half-light radii of the NQ-ETGs are shown as black arrows in Figure 8. These median sizes are equal to $4.3 \pm 0.4 \mathrm{kpc}$ and $8.7 \pm 0.9 \mathrm{kpc}$ in the low and high mass bins, respectively.

Franx et al. (2008) report a median size of $\sim 2 \mathrm{kpc}$ for Q-ETGs at $z=2.0$ in a mass bin equivalent to our lower mass bin. These high-redshift Q-ETGs are all likely to have been recently quenched at the time that we observe them. Within the large uncertainties involved, the change in size of the newly quenched galaxies is therefore consistent with the idea that NQETGs have, at fixed mass, a size that scales roughly as $(1+z)^{-1}$, i.e., with the cosmic scaling of the sizes of dark matter halos of a given mass. Equivalently, the mean stellar density of NQ-ETGs appears to scale roughly as the mean density of the universe.

This idea may then naturally explain the strong connection between SSFR and the mean stellar density of galaxies in the local universe. Quenched galaxies with low SSFR would naturally have higher stellar densities simply because they 

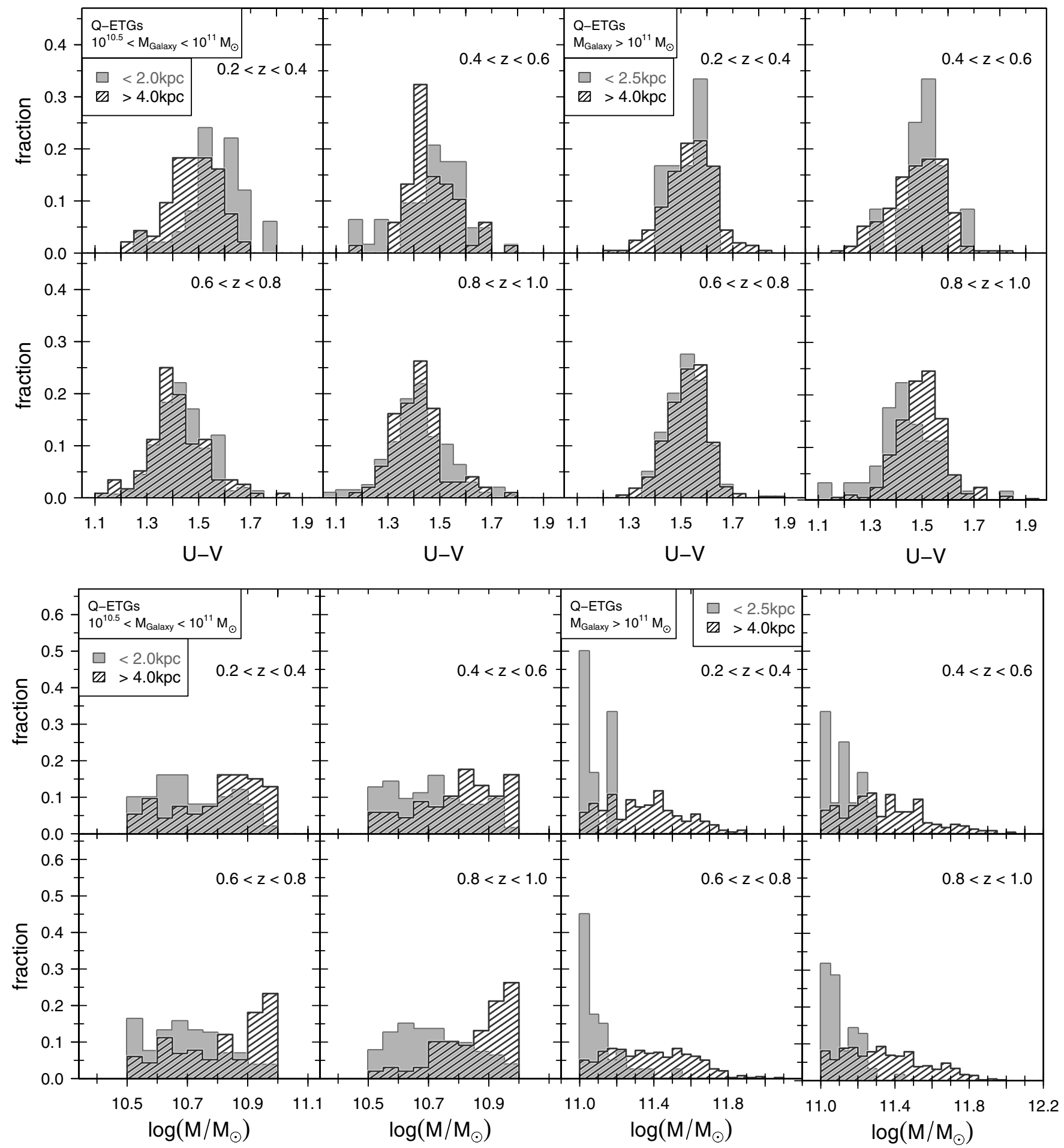

Figure 7. Top: the rest-frame $(U-V)$ color distributions of Q-ETGs in COSMOS, in the redshift interval $0.2<z<1$. Results are shown for two bins of stellar mass, $10^{10.5}<M_{\text {Galaxy }}<10^{11} M_{\odot}$ (two leftmost columns) and $M_{\text {Galaxy }}>10^{11} M_{\odot}$ (two rightmost column). The samples are split into the same four redshift intervals of Figure 4. Each panel compares the distributions of colors for Q-ETGs larger than $4 \mathrm{kpc}$ and smaller than $2 \mathrm{kpc}$ (or $2.5 \mathrm{kpc}$, in the low and high mass bins, respectively). Bottom: the corresponding stellar mass distributions for the compact and large samples of Q-ETGs of the top figure.

would, generally, have been quenched at significantly earlier epochs.

\subsection{Consistency with Predictions from a Continuity Equation}

We finally check whether the global number density growth factors that we have observed for the Q-ETGs between $z=1$ and $z=0.2$ are also roughly in agreement with those expected by applying a number continuity equation to the time evolution of the star-forming galaxy population, assuming that this is quenched without a substantial increase of stellar mass in the post-quenching phase (Peng et al. 2010). Anchoring their analysis to SDSS (York et al. 2007) and zCOSMOS (Lilly et al. 2007, 2009) data, these authors show strong evidence that, while "environment-quenching" dominates at lower masses, the dominant process that piles up galaxies at $>10^{10.5} M_{\odot}$ onto the 

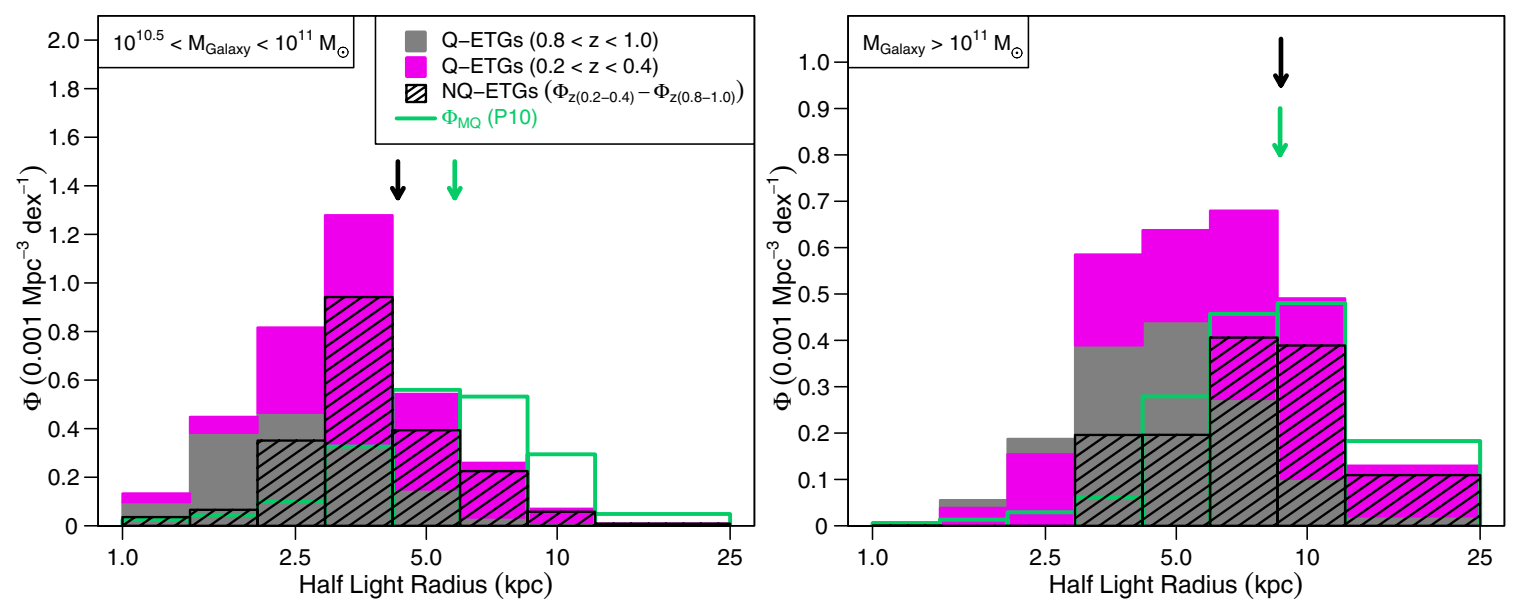

Figure 8. Empirical reconstruction for the size functions of NQ-ETGs over the $z \sim 1$ and $z \sim 0.2$ time period, defined through the difference between the $0.2<z<0.4$ (magenta histograms) and the $0.8<z<1.0$ (gray histograms) size functions of Q-ETGs of Figure 4. Top and bottom rows are respectively for $10^{10.5}<M<10^{11} M_{\odot}$ and $M_{\text {Galaxy }}>10^{11} M_{\odot}$ galaxies. The black arrows show the median sizes of these populations of NQ-ETGs over the $z=1 \rightarrow 0.2$ period (4.3 $\pm 0.4 \mathrm{kpc}$ and $8.7 \pm$ $0.9 \mathrm{kpc}$ in the low and high mass bins, respectively). In both mass bins, the green histograms show the size functions $\Phi_{\mathrm{MQ}}\left(r_{1 / 2}\right)$ of star-forming disks predicted to be mass-quenched between $z=1$ and $z=0.2$ according to the continuity equation of Peng et al. (2010; P10). The green arrows show the median sizes of these predicted mass-quenched galaxy populations $(5.8 \pm 0.1 \mathrm{kpc}$ and $8.7 \pm 0.1 \mathrm{kpc}$ in the low and high mass bins, respectively).

red-and-dead sequence is a quenching mechanism linked only to mass and not to environment (i.e., their "mass quenching"; cf. Figure 13 in Peng et al. 2010).

To explore whether mass quenching of star-forming galaxies is quantitatively consistent, in terms of numbers and sizes, with the evolution of the size function of Q-ETGs that we have observed, (1) we compute, in our two mass bins, the size (and $\Sigma_{\text {MASS }}$ ) functions for the star-forming population in the COSMOS data set and (2) we adopt the predictions of Peng et al. (2010) for sudden environment-independent mass quenching of star-forming galaxies, which is the relevant quenching mode at the mass scales of our sample.

We choose to limit this analysis to the star-forming disk galaxies in our COSMOS sample. We note that, at the stellar masses that we are investigating, disks represent $>80 \%$ of the star-forming population; furthermore, we also remark that, above $10^{10.5} M_{\odot}$, the population of star-forming disk galaxies is largely, $>90 \%$, made up of systems which have a substantial bulge component. In quantitative comparisons with previous studies, it is thus important to keep in mind that the star-forming population that we consider below is essentially a sample of starforming disks with a large if not dominant bulge component. We stress again, however, that we have tested that our results remain unchanged if the morphological selections are removed from our study. Apart from relatively small differences in the normalizations of the quenched and star-forming populations, which largely compensate each other, the only marginally detected difference is a higher number of large-size, irregular star-forming galaxies in the high relative to low redshift bin. This is not unexpected, and does not alter any of our conclusions. Our choice to select a morphologically well-behaved sample of starforming disks ensures a higher reliability in the estimates of galactic sizes relative to disturbed, irregular morphologies.

Figure 9 shows the size and $\Sigma_{\text {MASS }}$ functions of star-forming disk galaxies in the same redshift and mass bins as in Figure 4. Again we have chosen here a linear scale for $\Phi$ on the vertical axis, and error bars reflecting the Bayesian $1 \sigma$ CIs on the number density in each bin treated as a Poisson rate parameter. Note that star-forming galaxies do not disappear upon quenching, as they are continuously replenished from lower mass bins, thanks to star formation. Actually, they keep increasing their number density (except at the smallest sizes), as indeed found for their mass function both in Ilbert et al. $(2010,2013)$ and in the Peng et al. (2010) model.

The median half-light sizes of the low-mass (dashed curve) and high-mass (solid curve) star-forming population as a function of redshift are furthermore plotted as blue lines in the left panel of Figure 6 . Note that the star-forming disk galaxy population has typically an average median size $\left(\Sigma_{\text {MASS }}\right)$ a factor of 1.5-2 larger (lower) than that of Q-ETGs of similar mass and redshift. Also, the median size ( $\left.\Sigma_{\text {MASS }}\right)$ of star-forming disk galaxies shows, within the errors, a remarkable constancy with redshift over the $z=1$ to $z=0.2$ period. These results are in qualitative agreement with other work showing that the median sizes of star-forming galaxies are larger and evolve much slower than those of quenched galaxies (e.g., Franx et al. 2008). Quantitatively, however, these and other authors suggest a stronger evolution of the average size of star-forming galaxies than what we find. It is difficult to compare at these later redshifts results based on different samples: stellar masses may be systematically different, the nominal mass bins may differ from ours, and samples typically heterogeneous, i.e., they use different galaxy samples to compare different redshifts. For example, Franx et al. use, as several other authors, the SDSS sample to set the $z=0$ reference, with few data points at comparably high masses between $z=1$ and $z=0$. We thus refrain from attempting a direct comparison between our star-forming sample and others, and stress instead that, using the self-consistent COSMOS sample with uniformly derived sizes and well-defined morphological properties as described above, we actually detect a lack of evolution in the median size of $10>10^{10.5} M_{\odot}$ star-forming (mostly bulge-dominated) disk galaxies over the $z=1 \rightarrow 0.2$ redshift period. We defer to a follow-up analysis to understand the impact of cosmological disk fading and other factors on such apparent lack of evolution in the median size of disk star-forming galaxies in our sample.

We then use Equations (1) and (27) of Peng et al. (2010) to estimate the fractions of star-forming galaxies that are predicted to undergo mass quenching over the $z=1 \rightarrow 0.4$. Details of the derivation are described in Appendix D. For the lower mass bin, $10^{10.5}<M_{\text {Galaxy }}<10^{11} M_{\odot}$, this yields quenching fractions $f_{\mathrm{MQ}}$ of star-forming galaxies in the $0.8<z<1.0$, 

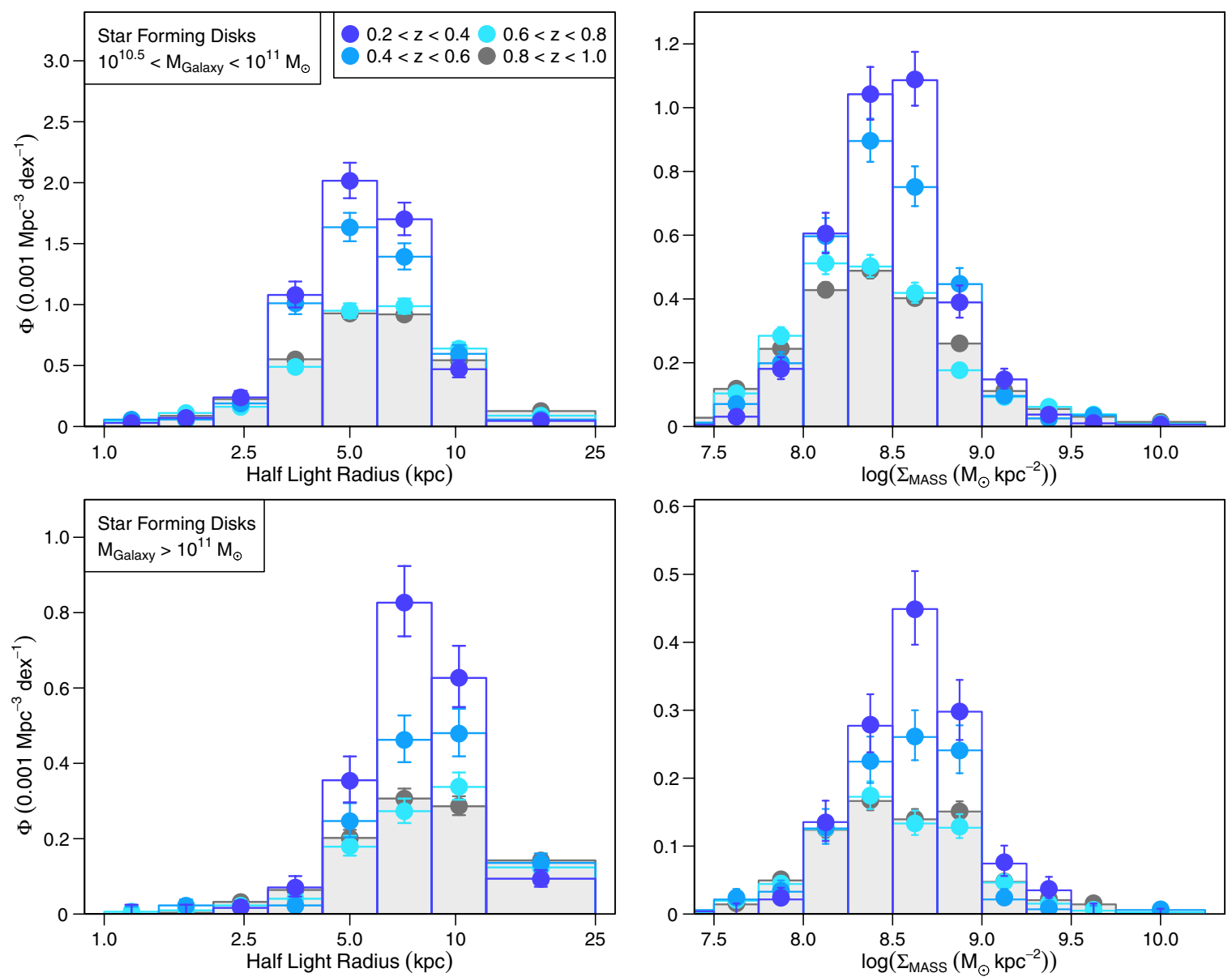

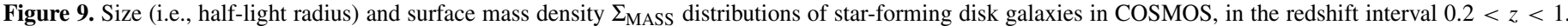

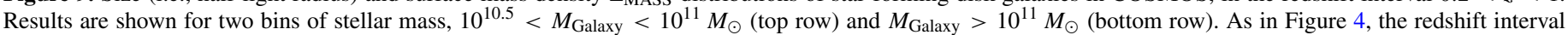

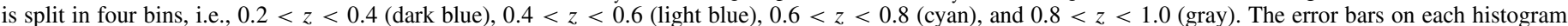

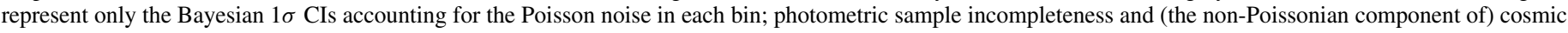

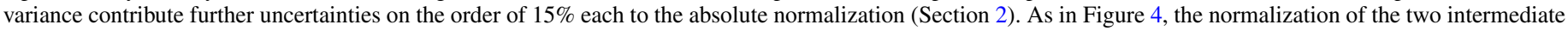
redshift bins is corrected for the known cosmic variance in the field (see the text).

$0.6<z<0.8$, and $0.4<z<0.6$ redshift bins of $0.22,0.16$, and 0.13 , respectively. At $M_{\text {Galaxy }}>10^{11} M_{\odot}$, the fractions of newly quenched galaxies in the same redshift bins are instead $f_{\mathrm{MQ}}=0.57,0.44$, and 0.35 , respectively.

We can thus finally compute the size function $\Phi_{\mathrm{MQ}}\left(M_{\mathrm{Galaxy}}\right.$, $r_{1 / 2}$ ) of star-forming galaxies of a given mass $M_{\text {Galaxy }}$ which should be mass-quenched and transferred to the Q-ETG sequence between $z=1$ and $z=0.2$ by summing up the size functions of star-forming galaxies of Figure 9 in the three highest redshift bins of our analysis, each weighted by its own mass-quenching fraction as given above, i.e.,

$$
\Phi_{\mathrm{MQ}}\left(r_{1 / 2}\right)=\sum_{i=1}^{3} f_{\mathrm{MQ}, i} \times \Phi_{\mathrm{SF}, i}\left(r_{1 / 2}\right)
$$

with $\Phi_{\mathrm{SF}, i}$ the size function of star-forming galaxies in the $i$ th redshift bin, and $i$ running through our three highest redshift bins. The results are presented in Figure 8 as green histograms; their medians are indicated by the green arrows, and are equal to $5.8 \pm 0.1 \mathrm{kpc}$ and $8.7 \pm 0.1 \mathrm{kpc}$ in the low and high mass bins, respectively. If mass quenching of star-forming galaxies of similar mass is responsible for the appearance of the largesize Q-ETG populations observed in both our mass bins, and if mass quenching does not change the sizes of the progenitors, these predicted distributions for the newly quenched galaxies should be similar to the corresponding empirical black hatched histograms of Figure 8.

At lower masses, $10^{10.5}<M_{\text {Galaxy }}<10^{11} M_{\odot}$, the comparison between black and green histograms readily shows a good agreement between the number densities integrated over all sizes of predicted and observed NQ-ETGs (respectively $2.1 \pm 0.2 \times 10^{-4} \mathrm{Mpc}^{-3}$ and $\left.2.6 \pm 0.2 \times 10^{-4} \mathrm{Mpc}^{-3}\right)$. At face value, however, the median size predicted for mass-quenched star-forming galaxies is larger by about $30 \%-40 \%$ relative to that derived for NQ-ETGs in the $z=1 \rightarrow 0.2$ period. This observed difference in the median sizes of the newly quenched remnants relative to their star-forming progenitors may be explained as an "apparent" (rather than physical) shrinkage, due to rapid fading of the disk components of these galaxies in the aftermath of their mass-quenching episode. This scenario is supported by the fact that, as we discuss above, $>90 \%$ of the star-forming progenitors are disk galaxies with a dominant bulge component. This implies that mass quenching efficiently retains the disk components of the star-forming progenitors, i.e., it has no direct effect of galaxy morphologies. This result is consistent with the morphological mix of $z<1$ quenched galaxies in COSMOS (e.g., Huertas-Company et al. 2013; Oesch et al. 2010), and with the analysis of the morphological mix of the quenched population in $z \sim 0$ virialized dark matter halos, which is found in ZENS (Carollo et al. 2012) to remain constant 
with halo-centric distance, despite the large increase of the total fraction of quenched satellites toward the halo centers (C. M. Carollo et al. 2013, in preparation).

Also at masses $M_{\text {Galaxy }}>10^{11} M_{\odot}$ there is an excellent agreement in the number densities, integrated between $z=1$ and $z=0.2$, of "predicted" and "observed" newly quenched galaxies in each individual size bin (and thus also in the total number densities integrated over all sizes, which are respectively $3.2 \pm 0.2 \times 10^{-4} \mathrm{Mpc}^{-3}$ and $3.1 \pm 0.5 \times 10^{-4} \mathrm{Mpc}^{-3}$ ). In addition, at these high masses, the median size for the predicted mass-quenched population of star-forming disk galaxies over the $z=1 \rightarrow 0.2$ time span (green histogram) is strikingly similar to that inferred, over the same redshift range, for the newly quenched population of Q-ETGs (black histogram). This means that, if also at these high masses mass-quenching of progenitor star-forming disk galaxies drives the average size evolution of the QETG population, the physical mechanism behind mass quenching should conserve stellar mass and size, while transforming morphologies from disk-like to spheroidallike. At least in part, this could be explained again by fading of the disk components of the progenitor bulge-dominated starforming galaxies, since at such high masses these progenitors are likely to have high bulge-to-disk ratios and thus small disk components of sizes comparable to those of the dominant bulges. As already commented above, however, at these high masses other factors such as individual galaxy growth and merging are also likely to contribute to the evolution of the Q-ETG population, as indicated by the detected $~ 30 \%-40 \%$ decrease toward lower redshifts in the number density of the most compact of such systems, and by the similarity in restframe colors of small and large galaxies.

\section{SUMMARY AND CONCLUSIONS}

In the previous sections, we have argued that the apparent size evolution of the Q-ETG population is largely due to the appearance of new, larger, galaxies adding to a rather stable pre-existing population of more compact galaxies which has a more or less constant number density. We do not claim that this is the only effect in operation, but it is certainly in our view the dominant one.

This conclusion is most strongly supported in the lower mass bin of Q-ETGs that we have considered in this paper, $10^{10.5}<M_{\text {Galaxy }}<10^{11} M_{\odot}$, which samples the bulk of the quenched early-type population. At these masses, our data show (1) no evolution in the number density of the smallest and densest Q-ETGs between $z=1$ and $z=0.2$, and (2) evidence of a strong buildup in the population of their larger siblings over the same redshift range. In other words, at these masses, Q-ETGs roughly have a constant size, once formed, and newly formed Q-ETGs are being added at larger sizes. Previous works had suggested that Q-ETGs evolve mildly in size, once formed, and that the evolution of the mass-size relation is also driven by newly formed Q-ETGs being added at larger sizes (e.g., Valentinuzzi et al. 2010; Cassata et al. 2011; Newman et al. 2012; Poggianti et al. 2013). Franx et al. (2008) found that galaxies with a surface density of $\sim 10^{9} M_{\odot} \mathrm{kp}^{-2}$ are "red and dead" at low redshift and almost all are star forming by $z=2$, also evidence for a late evolution of galaxies onto the red sequence. Our results put on a firm foundation and quantify these earlier suggestions, and show that, especially at sub- $M^{*}$ masses, it is the addition of newly formed Q-ETGs at larger sizes rather than the growth of individual galaxies that dominates the evolution of the mass-size relation.
The alternative interpretation that galaxies are individually expanding in size while the new ones are added to replace them at the small end of the size distribution runs counter to our finding that (1) the compact quenched population shows increasingly redder $(U-V)$ colors toward lower redshifts, with an average color difference between $z=1$ and $z=0.2$ that is well consistent with the aging, over this time span, of a passively evolving galaxy population; and (2) that the rest-frame $(U-V)$ colors of the larger Q-ETG are systematically bluer (as also suggested at the higher and lower redshifts respectively by Saracco et al. 2011 and van der Wel et al. 2009), pointing to a more recent quenching than the corresponding compact population. Together this evidence excludes the possibility that the stable number density between $z=1$ and $z=0.2$ of compact Q-ETGs could be due to a balance between the formation rate of new compact Q-ETGs and their depletion rate due to a size growth out of the compact bin, and indicates that the newly quenched galaxies are being added at the larger end of the size distribution.

The growth of the median radius of the quenched earlytype population has usually been interpreted in terms of the evolution of individual galaxies (e.g., Oser et al. 2012), without considering the changes in the number density that, as argued here, indicate that the effect is driven by the addition of newly quenched larger galaxies at later epochs. The most popular mechanism has been size increase through minor merging (e.g., Hopkins et al. 2009; Feldmann et al. 2010; Cimatti et al. 2012). This latter scenario for the growth of the median size of Q-ETGs requires however about 10 mergers with about 1:10 mass ratios to explain the observed size growth since $z \sim 2$ (e.g., van de Sande et al. 2013, and references therein). None of these minor mergers can be gas-rich, since it is believed that gas infall toward the primary galaxy center would lead to nuclear star formation and thus to a "shrinkage" of its half-light radius. Such a sequence of 10 dry mergers with no intervening wet mergers seems unlikely, especially on account of the fact that, at the $\sim 10^{10} M_{\odot}$ mass scale of the 1:10 companions of $\sim 10^{11} M_{\odot}$ galaxies, gas-rich systems largely outnumber their dry counterparts. This adds to another difficulty of this scenario, namely, the dearth of suitable nearby companions highlighted by Newman et al. (2012).

Nevertheless, it is clear that some such individual size evolution must have happened (although our data show that it is not the dominant driver of the size evolution of the population). Not least, our own analysis has indicated a modest decrease in the number density of compact $r_{1 / 2}<2 \mathrm{kpc}$ galaxies at $M_{\text {Galaxy }}>10^{11} M_{\odot}$ by $\sim 30 \%-40 \%$ over the redshift interval $z=1 \rightarrow 0.2$. These very massive galaxies also show weaker color trends; we highlight here, as an open issue, to understand the similarity between the average rest-frame colors of compact and large Q-ETGs with masses above $M^{*}$.

A fact to keep in mind is that quenched galaxies that are as massive as $10^{11} M_{\odot}$ and above already by redshift $z \sim 1$ will typically be the central galaxies in halos that have a mass today of $\geqslant 10^{13} M_{\odot}$. Hydrodynamical simulations in a $\Lambda$-CDM universe show that, over this period, such galaxies grow in stellar mass mostly through minor mergers; these mergers produce outer halos and leave the central density of galaxies almost constant (e.g., Hopkins et al. 2009; Feldmann et al. 2010). We also note that in the continuity analysis of Peng et al. (2010), $10^{11} M_{\odot}$ was identified as a threshold above which post-quenching mass increase through mergers was likely to be significant, and below which it was generally unlikely to have been. The $30 \%$ decrease 

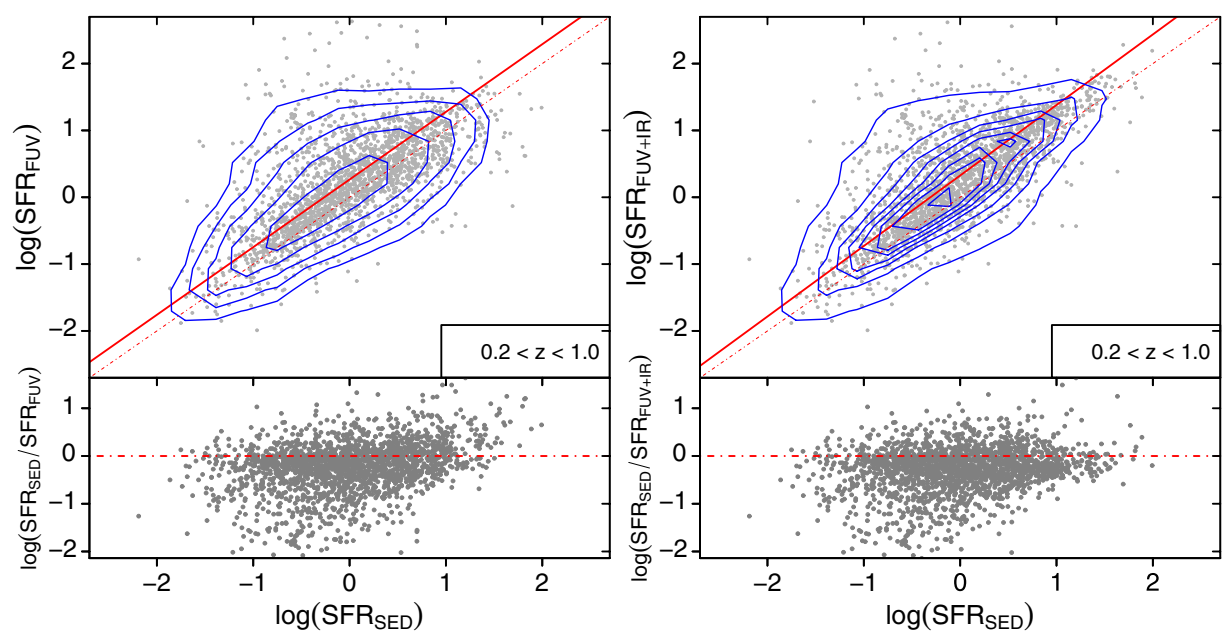

Figure 10. Left panel: fit to the relation between UV-based, dust-corrected SFRs and corresponding values derived from SED fits to galaxy photometric points. Right panel: fit to the relation between the sum of IR-based and dust-uncorrected UV-based SFRs, and SFRs derived from SED fits to galaxy photometric points. The good correlation between $\mathrm{SFR}_{\mathrm{FUV}}$, cor and $\mathrm{SFR}_{\mathrm{IR}+\mathrm{UV}}$ with $\mathrm{SFR}_{m}$ motivates our use of the latter, which is available for the whole $I_{814 W}<24$ COSMOS sample. Note that only one out of 25 points is plotted, for clarity of presentation. The contours refer, however, to the whole sample (with each line referring to an increase in number density of a factor of two).

in density found here, and possibly the colors, of these very massive Q-ETGs is thus consistent with a picture in which a fraction of them accrete diffuse halos at later times, as also suggested by many other studies (see quoted references). We re-emphasize, however, that even at this high mass end the dominant effect in the increase of the average radius for the whole population remains the appearance, at later epochs, of equally massive but larger (in half-light size) Q-ETGs.

The present analysis indicates that the typical sizes of NQ-ETGs increase with time, i.e., that quenching of progressively larger star-forming galaxies progressively outnumbers that of galaxies with smaller sizes (which dominated the quenching rate at earlier epochs); this is the dominant cause of the apparent increase in size of the integrated quiescent population. The typical sizes of these newly quenched galaxies appear to scale as roughly $(1+z)^{-1}$, suggesting that the mean stellar density within these newly quenched galaxies scales as the mean density of the universe at the time of quenching. This idea may unify a number of important observational facts, including the observed correlation between SSFR and mean stellar density in the local universe.

T.B., A.C., E.C., and M.O. thank the Swiss National Science Foundation for financial support. A.R. acknowledges the ETHZ Institute of Astronomy for its kind hospitality at the time when this research was started and this paper first laid down, and the National Institute for Astrophysics for support through the grant INAF-PRIN 2010.

\section{APPENDIX A}

\section{THE CHOICE OF SED-BASED SFRs}

Before adopting as fiducial SFRs those derived from the SED fits, we analyzed in detail SFR estimates derived using (1) dust-extinction-corrected rest-frame far-UV (FUV) fluxes, using the Calzetti et al. (2000) extinction curves together with the reddening relation $E(B-V)=0.23 \beta+0.49$ of Meurer et al. (1999), as well as the Wijesinghe et al. (2011) reddening relation $E(B-V)=0.2 \beta+0.33$ (with $\beta$ the slope of the UV continuum in a power-law approximation); (2) Spitzer/MIPS $24 \mu \mathrm{m}$ detections down to a limiting flux of $80 \mu \mathrm{Jy}$ with Dale \&
Helou (2002) templates (MIPS catalog courtesy of E. Le Floch); and (3) the combination of IR-based and FUV-based SFRs, the latter not corrected for extinction.

Given the flux limits of the available FUV and Spitzer COSMOS photometry, the FUV- or $24 \mu \mathrm{m}$ based SFRs result in strong and redshift-dependent selection biases toward mass and SFR. The FUV selection picks up 75\% of galaxies at the redshifts of interest, but it misses the most massive and quiescent systems (median mass of the FUV-detected galaxies is $\log \left(M / M_{\odot}\right)=9.4$, compared with a median mass of the galaxies not detected in FUV of $\left.\log \left(M / M_{\odot}\right)=9.9\right)$. Infraredbased SFRs are only available for about $17 \%$ of the COSMOS galaxies and pick up only the most massive star-forming objects (IR median mass $<\log M / M_{\odot}=10.3$, compared to 9.3 for galaxies not detected in the MIPS images in the $0.2<z<1.0$ range), with a rapidly increasing SFR detection threshold as a function of redshift.

The comparison of the extinction-corrected FUV-based SFRs with the SFRs derived by summing the uncorrected-FUV plus IR contributions, favors the Meurer et al. reddening relation. Comparison of the FUV SFRs, corrected for extinction using this relation, shows a small shift but, overall, an excellent agreement with the SFRs derived from our SED fits. A fit of the relation gives $\log \left(\mathrm{SFR}_{\mathrm{FUV}, \text { cor }}\right)=1.01 \pm 0.01 \times \log \left(\mathrm{SFR}_{\mathrm{SED}}\right)+$ $0.27 \pm 0.01$, with a formal $1 \sigma$ scatter of 0.48 (see left panel of Figure 10). A similar fit to the relation between SEDbased SFRs and SFRs obtained by summing IR-based and (dust-uncorrected) UV-based values gives $\log \left(\mathrm{SFR}_{\mathrm{FUV}+\mathrm{IR}}\right)=$ $1.06 \pm 0.01 \times \log \left(\mathrm{SFR}_{\mathrm{SED}}\right)+0.33 \pm 0.01$, with a formal $1 \sigma$ scatter of 0.45 (see right panel of Figure 10). These excellent agreements validate our adoption of the SED-based SFRs for our analysis, which have the virtue of being uniformly derived and available for all galaxies in our sample. We stress, however, that we have tested that our results do not depend on the choice of SFR diagnostic adopted for this analysis.

\section{APPENDIX B}

\section{RELIABILITY OF THE CORRECTION FUNCTIONS FOR GALAXY SIZES: CONSISTENCY CHECKS}

Simple analyses and considerations using the uncorrected and corrected (with the corrections of Section 3) magnitude-size 

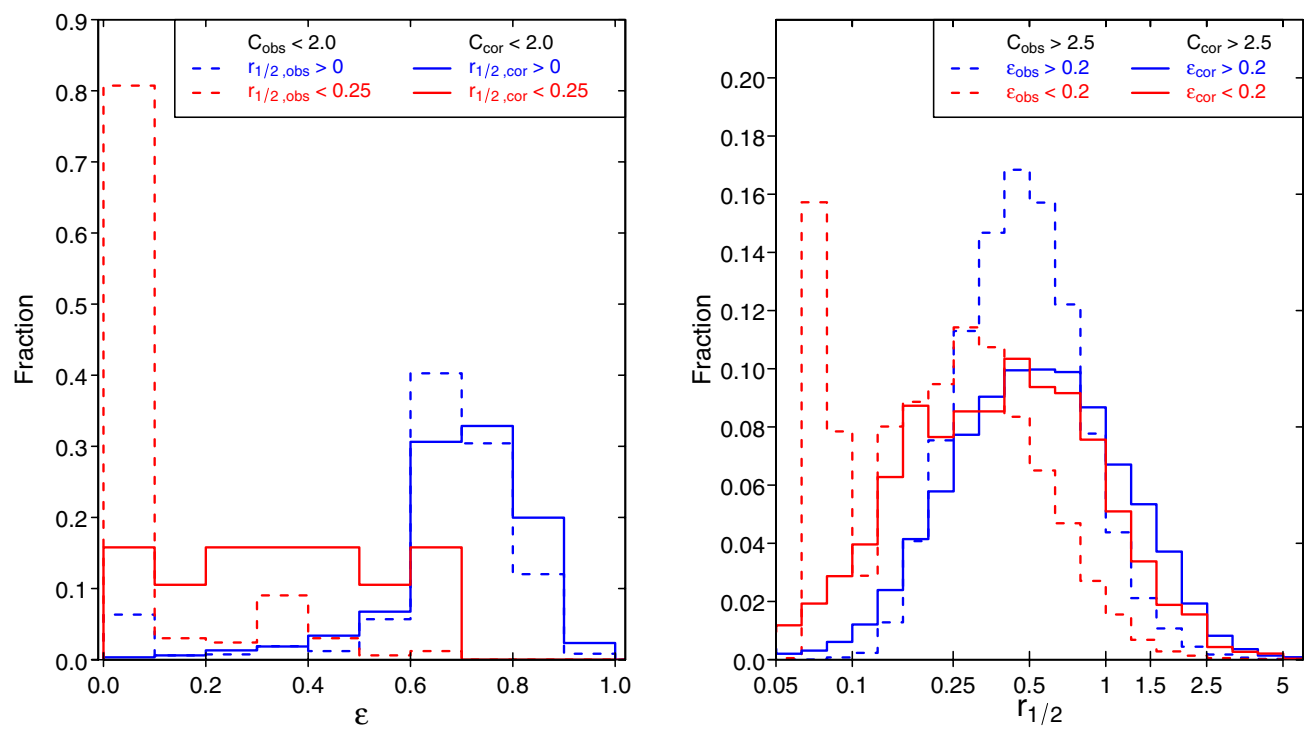

Figure 11. Left panel: the uncorrected (dashed line) and corrected (solid line) distribution of ellipticities for $C<2$ disk galaxies in COSMOS. Blue is for the whole $C<2$ sample, red is for the subsample of $r_{1 / 2}<0$ '.25 galaxies. Right panel: the uncorrected (dashed) and corrected (solid) sizes distributions of $C>2.5$ disk galaxies in COSMOS. All histograms are normalized to the total number of galaxies in the plotted samples. Both panels show that systematics in the raw size measurements are substantially reduced after applying the corrections of Section 3.

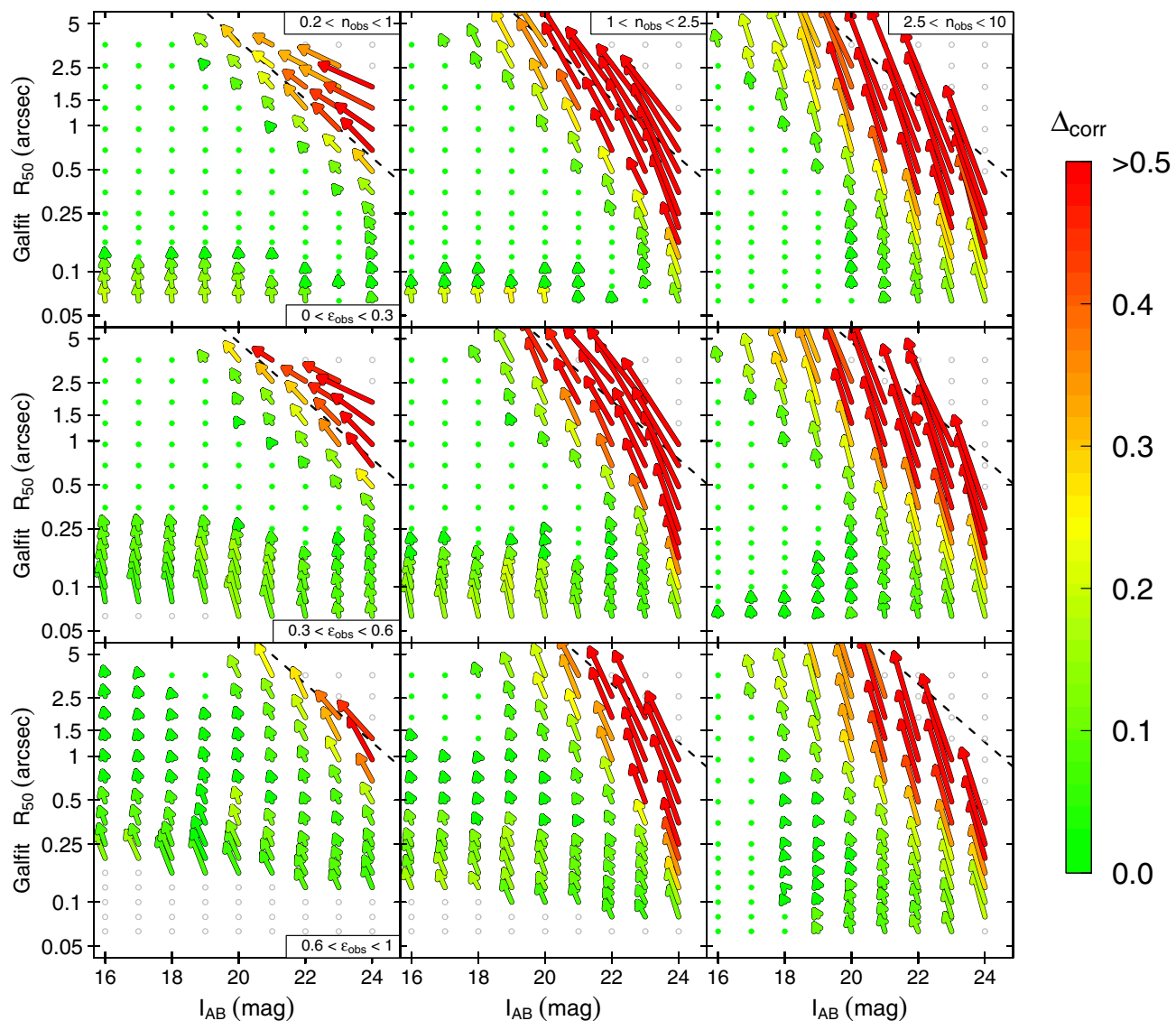

Figure 12. Correction grid for observed half-light radius and magnitude values for $I_{814 \mathrm{~W}}<24$ mag COSMOS galaxies, as already presented in Figure 2, but this time for size and magnitude measurements obtained through GALFIT fits to the galaxies' surface brightness distributions. The three bins of concentration index of Figure 2 are here replaced by three bins in Sérsic index $n$. Colors and symbols are as in Figure 2.

distributions show the importance of accounting for systematic biases in Kron-style size measurements.

The left panel of Figure 11 shows the distributions of uncorrected and corrected ellipticities of galaxies with concentration values $C<2$, for the entire sample (blue) and the sample limited by $r_{1 / 2}<0.25$ arcsec. Uncorrected measurements would lead to an unphysical dependence of sizes of small disk on inclination/viewing angle, as evident from the figure. The correction functions restore, from the low ellipticity bin to the high ellipticity bin, a fraction of intrinsically small, edge-on disks that were rounded/blurred by the observational PSF, and furthermore recover their intrinsic sizes, which were reduced by this PSF blurring. The right panel in the same figure shows the uncorrected and corrected size distributions of $C>2.5$ galaxies, 


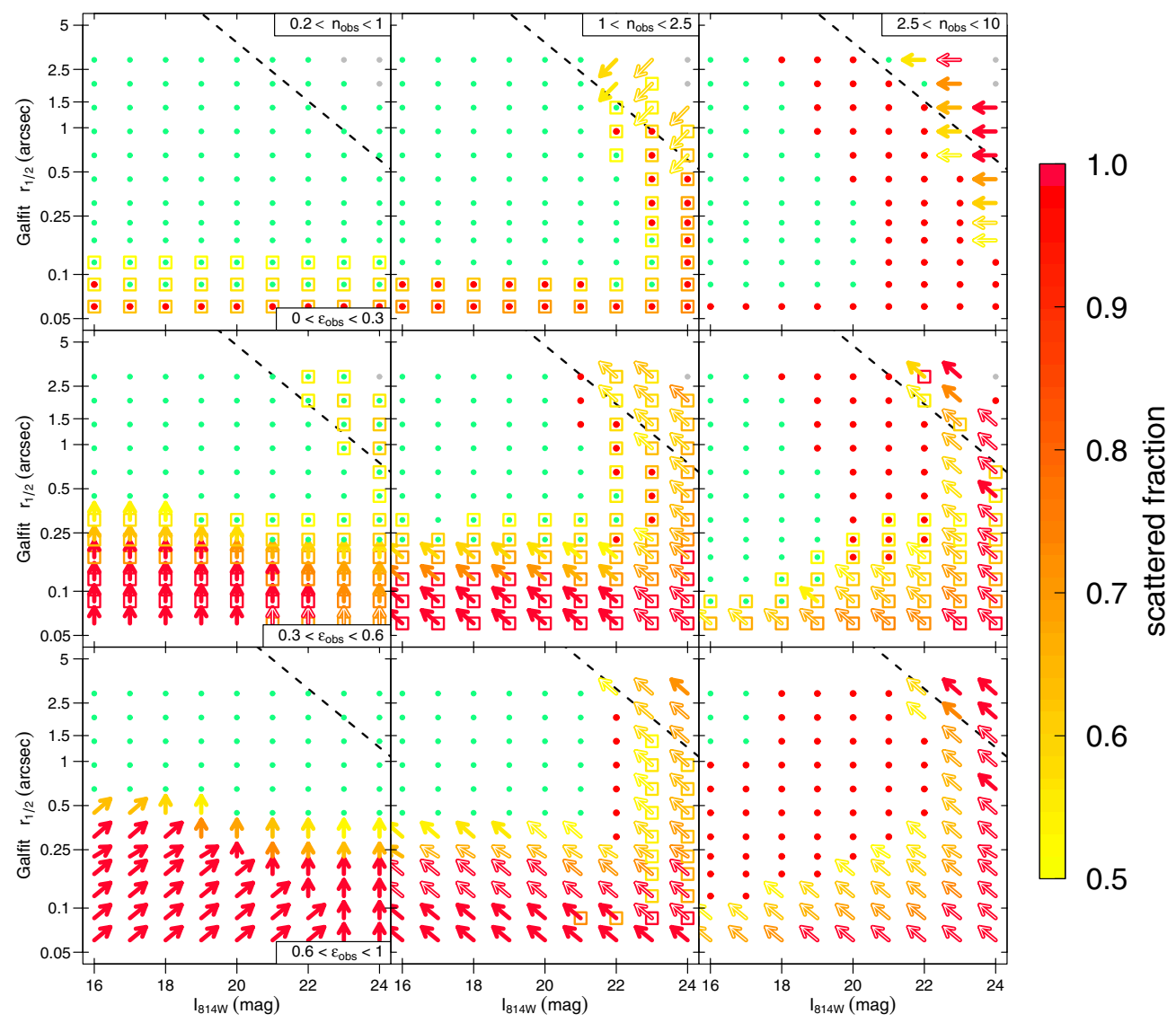

Figure 13. Analysis of scattering of model galaxies in and out of true/input ellipticity and concentration bins, as in Figure 3, but this time for measurements obtained through GALFIT fits to the galaxies' surface brightness distributions. Similarly to Figure 12, the three bins of concentration index of Figure 3 are here replaced by three bins in Sérsic index $n$. Colors and symbols are as in Figure 3.

mostly spheroids and bulge-dominated disks, in two bins of ellipticity, above and below $\epsilon=0.2$. Results are again shown in red and blue for uncorrected and corrected quantities, respectively. A significant fraction of intrinsically high ellipticity, $C>2.5$ galaxies which our morphological analysis and inspection confirm to be primarily edge-on, bulge-dominated disks, are returned by the correction functions to the $e_{\text {cor }}>0.2$ bin. Furthermore, the size distributions of round and inclined systems are in much better agreement after the application of the corrections.

\section{APPENDIX C}

\section{THE NEED FOR CORRECTING SIZES DERIVED FROM SURFACE BRIGHTNESS FITS: A SHOWCASE WITH GALFIT}

As highlighted in Section 3.2 it is not only (Kron, Petrosian, or isophotal) aperture-based measurements which require a correction function to aid the recovery of true galaxy magnitudes, sizes, ellipticities, and concentration indices from their raw (i.e., observationally biased) counterparts. In this Appendix, we illustrate the nature of these biases for the case of Sérsic (1963) profile $^{14}$ modeling with GALFIT (Peng et al. 2002) using a

\footnotetext{
14 The well-known Sérsic radial profile is defined as $\mu(r)=$ $\mu_{0} \exp \left[-(r / \alpha)^{1 / n}\right]$, where $\mu(r)$ is the intensity at radius $r$ and $\mu_{0}$ is the intensity at the galaxy center, i.e., in $r=0 . \alpha$ is the scale length, i.e., the radius at which the intensity drops by a factor $e^{-1}$. The parameter $n$ is the "Sérsic index," and controls the degree of central concentration, i.e., the shape of the profile.
}

process of artificial galaxy simulation equivalent to that introduced for the calibration of the SExtractor/ZEST+ measurements that we adopt in our analysis. Specifically, we began by generating $>800,000$ artificial galaxy images with input parameters randomly drawn within the following limits: $19<I_{814 \mathrm{~W}}<$ $25 \mathrm{mag}, 0.05<r_{1 / 2}<2.5$ arcsec, $0<e<0.95$, and Sérsic index $0.2<n<8$. As before each model galaxy was constructed at a pixel scale of 0.03 arcsec and convolved with a representative ACS $I_{814 W}$ PSF (Rhodes et al. 2007), and added with Poisson noise to an empty region of sky from the real COSMOS HST/ACS imaging. SExtractor was then run to generate object masks and to estimate starting values for use in GALFIT. In particular, we adopt the Kron magnitude, the mean isophotal ellipticity and position angle, and the Kron half-light radius as initial guesses for their model counterparts-and all fits were begun with an initial guess of $n=2.5$ for the Sérsic index. After running GALFIT the output parameters of all convergent fits (i.e., those which did not end in a catastrophic error causing the program to crash) were saved for comparison against our input model parameters. The results of this analysis are presented in Figures 12 and 13 (analogs to Figures 2 and 3, respectively).

Examination of the "correction vectors" diagram for the GALFIT simulations shows two important differences relative to the analog diagram for the SExtractor/ZEST+ case shown in Figure 2: (1) not surprisingly, the incorporation of the observational PSF into the fitting algorithm GALFIT does indeed outperform SExtractor/ZEST+ in size recovery for the most compact galaxies; however, (2) in the low surface brightness regime, the GALFIT-based measurement are substantially 

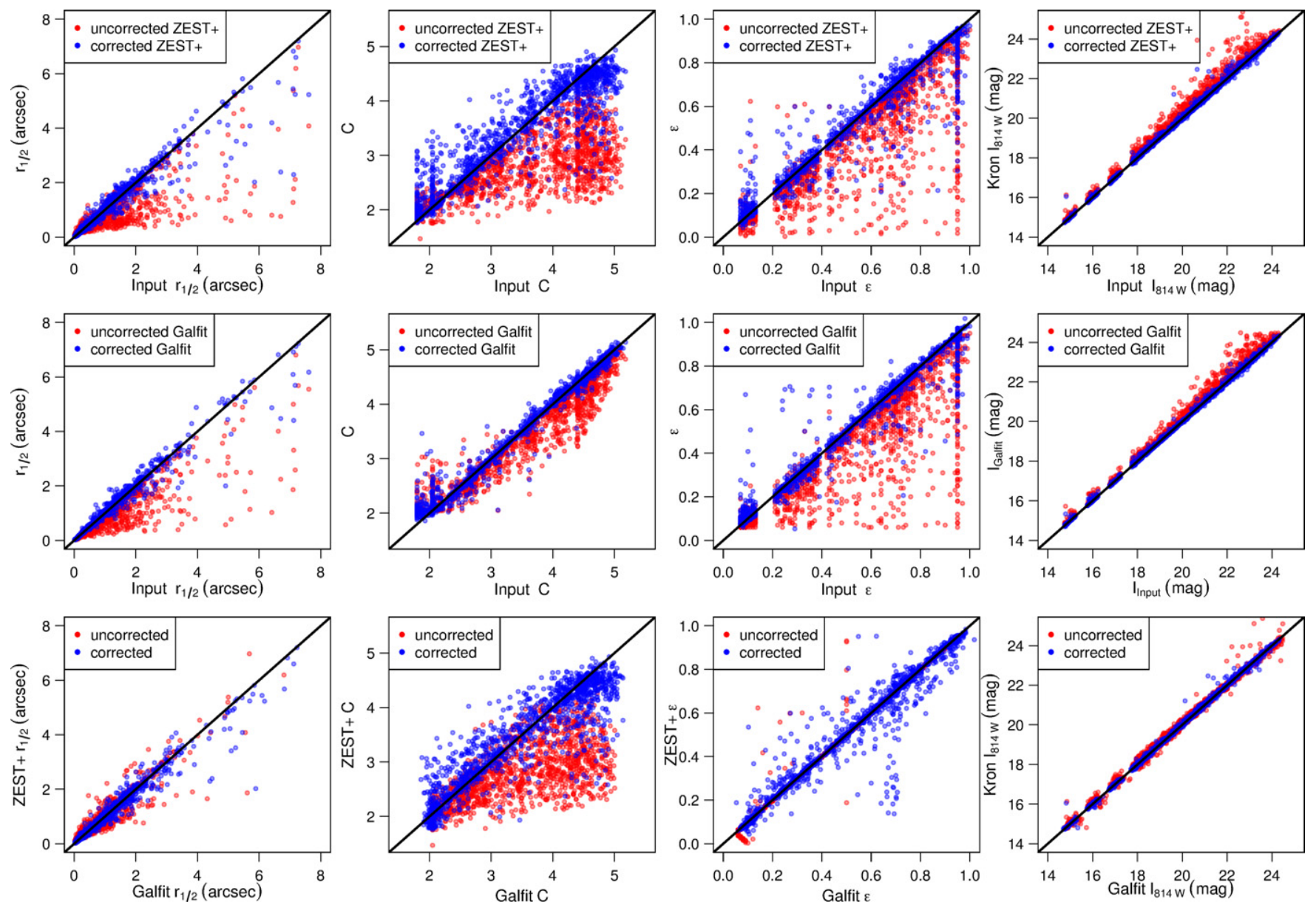

Figure 14. Comparison between uncorrected (red points) and corrected (blue points) parameters obtained with ZEST+ (top row) and GALFIT (middle row). Corrected parameters are obtained by applying the calibrations discussed in Section 3.2. The shown parameters are, from left to right: half-light radius $r_{1 / 2}$, concentration $\mathrm{C}$, ellipticity $\epsilon$, and $I_{814 \mathrm{~W}}$ magnitude. For clarity, the plot shows results for only 1500 of the simulated galaxies. The bottom row shows the comparison between measurements obtained with ZEST+ and GALFIT before (red) and after (blue) the application of the corrections. The applied calibrations substantially improve the scatter between "true" (input) and "observed" values, and lead to a very good agreement between corrected measurements obtained with either the ZEST+ aperture approach or the GALFIT surface brightness fitting approach.

affected by systematic offsets from their true (input) values - with a clear trend toward underestimation of both apparent magnitudes and sizes. Note also that, as indicated by the color coding of the error vectors in Figures 12 and 13, the random errors in size recovery of high concentration $/ n$-Sérsic systems with GALFIT are worse in the low surface brightness regime than they are for the more stable Kron approach (as implemented in SExtractor/ZEST+). Corrections to model-based measurements may be important especially at high redshifts, where faint disk components may populate the low surface brightness regime of the correction diagrams.

Once the GALFIT- and Kron-based size measurements are properly corrected for, as we do in this paper, then both estimates obtained with either SExtractor/ZEST+ or GALFIT are in excellent agreement, as shown in Figure 14. The figure shows the inherent consistency of our correction functions through a comparison of the apparent magnitudes and sizes of 30 randomly drawn high Sérsic- $n$ COSMOS galaxies, recovered after applying our correction maps to both the SExtractor/ZEST+ and the GALFIT measurements. Although both measurement techniques require different and, in both cases, non-negligible adjustments against systematic recovery biases in this parameter space, the resulting distributions are in very good agreement, with only small random offsets between the two sets of estimates.
This gives us confidence that the results of this study are robust against the choice of size measurement algorithm owing to the use of our correction function approach.

\section{APPENDIX D THE QUENCHED FRACTIONS FROM
PENG ET AL. (2010)}

To compare the Peng et al. predictions with our estimates, we convert the mass- and time-dependent evolution of the sSFR in their Equation (1),

$$
\operatorname{sSFR}\left(M_{\text {Galaxy }}, t\right)=2.5\left(\frac{M_{\text {Galaxy }}}{10^{10} M_{\odot}}\right)^{\beta}\left(\frac{t}{3.5 \mathrm{Gyr}}\right)^{-2.2} \mathrm{Gyr}^{-1}
$$

(with $\beta=-0.1$, see references above), into the mass- and timedependent evolution of the reduced specific star formation rate, $\operatorname{rsSFR}\left(M_{\text {Galaxy }}, t\right)$, as done in Lilly et al. (2013); this is necessary since, in contrast with those authors (who use the "actual" stellar mass trapped in long-lived stars in their study), we defined the stellar mass as equal to the integral of the SFR (see Section 2.2). We have

$$
\operatorname{rsSFR}\left(M_{\text {Galaxy }}, t\right)=(1-R) \times \operatorname{sSFR},
$$


where $R$ is the fraction of the baryonic mass that is converted into stars and is promptly returned to the interstellar medium (with the remaining fraction $1-R$ remaining locked in longlived stars). The inverse of the rsSFR sets the characteristic timescale for the buildup of the long-lived stellar population. As in Lilly et al. (2013), we assume an instantaneous mass return and $R=0.4$, as derived from stellar population models of Bruzual \& Charlot (2003).

The fraction $f_{\mathrm{MQ}}$ of transient, just being-quenched objects at any time $t$ can be derived from Equation (27) of Peng et al. (2010), modified to take into account Equation (D2) above, by computing

$$
f_{\mathrm{MQ}}(t)=\frac{\Phi_{\mathrm{SF}}(t) \times \operatorname{rsSFR}\left(M_{\mathrm{Galaxy}}, t\right) \times \frac{M_{\text {Galaxy }}}{M^{*}}}{\Phi_{\mathrm{SF}}(t)},
$$

with $\Phi_{\mathrm{SF}}(t)$ being the number density of star-forming galaxies at the same epoch. We can thus predict the fractions $f_{\mathrm{MQ}, i}$ of newly quenched galaxies in each redshift bin $i$ of our analysis as

$$
f_{\mathrm{MQ}, i}=\left.f_{\mathrm{MQ}}\right|_{t\left(z_{\mathrm{high}, i}\right)} ^{t\left(z_{\text {low }}\right)}=\frac{M_{\mathrm{Galaxy}}}{M^{*}} \int_{t\left(z_{\mathrm{high}, i}\right)}^{t\left(z_{\text {low }, i}\right)} \operatorname{rsSFR}\left(M_{\mathrm{Galaxy}}, t\right) d t,
$$

with $z_{\text {high, } i}$ and $z_{\text {low }, i}$ being the upper and lower limit of the $i$ th redshift bin.

\section{REFERENCES}

Abazajian, K. N., Adelman-McCarthy, J. K., Agüeros, M. A., et al. 2009, ApJS, 182,543

Barro, G., Faber, S. M., Pérez-González, P. G., et al. 2013, ApJ, 765, 104

Bertin, E., \& Arnouts, S. 1996, A\&AS, 117, 393

Brammer, G. B. 2009, ApJL, 706, L173

Bruzual, G., \& Charlot, S. 2003, MNRAS, 344, 1000

Bundy, K., Scarlata, C., Carollo, C. M., et al. 2010, ApJ, 719, 1969

Calzetti, D., Armus, L., Bohlin, R. C., et al. 2000, ApJ, 533, 682

Cameron, E., \& Driver, S. P. 2007, MNRAS, 377, 523

Cameron, E., Carollo, C. M., Oesch, P., et al. 2010, MNRAS, 409, 346

Capak, P., Aussel, H., Ajiki, M., et al. 2007, ApJS, 172, 99

Cardelli, J. A., Clayton, G. C., \& Mathis, J. S. 1989, ApJ, 345, 245

Carollo, C. M., Cibinel, A., Lilly, S. J., et al. 2012, arXiv:1206.5807

Cassata, P., Giavalisco, M., Guo, Y., et al. 2011, ApJ, 743, 96

Chabrier, G. 2003, ApJL, 586, L133

Cibinel, A., Carollo, C. M., Lilly, S. J., et al. 2012, arXiv:1206.6496

Cimatti, A., Cassata, P., Pozzetti, L., et al. 2008, A\&A, 482, 21

Cimatti, A., Daddi, E., \& Renzini, A. 2006, A\&A, 453, L29

Cimatti, A., Nipoti, C., \& Cassata, P. 2012, MNRAS, 422, L62

Coleman, G. D., Wu, C.-C., \& Weedman, D. W. 1980, ApJS, 43, 393

Covington, M. D., Primack, J. R., Porter, L. A., et al. 2011, MNRAS, 415, 3135

Daddi, E., Renzini, A., Pirzkal, N., et al. 2005, ApJ, 626, 680

Dale, D. A., \& Helou, G. 2002, ApJ, 576, 159

Domínguez Sánchez, H., Pozzi, F., Gruppioni, C., et al. 2011, MNRAS, 417, 900

Dullo, B. T., \& Graham, A. W. 2013, ApJ, 768, 36

Elmegreen, B. G., Elmegreen, D. M., Fernandez, M. X., \& Lemonias, J. J. 2009, ApJ, 692, 12

Feldmann, R., Carollo, C. M., Mayer, L., et al. 2010, ApJ, 709, 218

Feldmann, R., Carollo, C. M., Porciani, C., et al. 2006, MNRAS, 372, 565

Franx, M., van Dokkum, P. G., Schreiber, N. M. F., et al. 2008, ApJ, 688,770
Giavalisco, M., Ferguson, H. C., Koekemoer, A. M., et al. 2004, ApJL, 600, L93

Graham, A. W., Driver, S. P., Petrosian, V., et al. 2005, AJ, 130, 1535

Guo, Y., Giavalisco, M., Cassata, P., et al. 2011, ApJ, 735, 18

Häussler, B., McIntosh, D. H., Barden, M., et al. 2007, ApJS, 172, 615

Hopkins, P. F., Bundy, K., Hernquist, L., Wuyts, S., \& Cox, T. J. 2010, MNRAS, 401, 1099

Hopkins, P. F., Bundy, K., Murray, N., et al. 2009, MNRAS, 398, 898

Huertas-Company, M., Mei, S., Shankar, F., et al. 2013, MNRAS, 428, 1715

Ilbert, O., Capak, P., Salvato, M., et al. 2009, ApJ, 690, 1236

Ilbert, O., McCracken, H. J., Le Fevre, O., et al. 2013, arXiv:1301.3157

Ilbert, O., Salvato, M., Le Floc'h, E., et al. 2010, ApJ, 709, 644

Kinney, A. L., Calzetti, D., Bohlin, R. C., et al. 1996, ApJ, 467, 38

Koekemoer, A. M., Aussel, H., Calzetti, D., et al. 2007, ApJS, 172, 196

Kraft, R. P., Burrows, D. N., \& Nousek, J. A. 1991, ApJ, 374, 344

Kron, R. G. 1980, ApJS, 43, 305

Leauthaud, A., Massey, R., Kneib, J.-P., et al. 2007, ApJS, 172, 219

Lilly, S. J., Carollo, C. M., Pipino, A., Renzini, A., \& Peng, Y. 2013, arXiv: 1303.5059

Lilly, S. J., Le Brun, V., Maier, C., et al. 2009, ApJS, 184, 218

Lilly, S. J., Le Fèvre, O., Renzini, A., et al. 2007, ApJS, 172, 70

Mancini, C., Daddi, E., Renzini, A., et al. 2010, MNRAS, 401, 933

McCracken, H. J., Capak, P., Salvato, M., et al. 2010, ApJ, 708, 202

McGrath, E. J., Stockton, A., Canalizo, G., Iye, M., \& Maihara, T. 2008, ApJ, 682,303

Meurer, G. R., Heckman, T. M., \& Calzetti, D. 1999, ApJ, 521, 64

Naab, T., Johansson, P. H., \& Ostriker, J. P. 2009, ApJL, 699, L178

Newman, A. B., Ellis, R. S., Bundy, K., \& Treu, T. 2012, ApJ, 746, 162

Nipoti, C., Treu, T., Auger, M. W., \& Bolton, A. S. 2009, ApJL, 706, L86

Oesch, P. A., Carollo, C. M., Feldmann, R., et al. 2010, ApJL, 714, L47

Onodera, M., Daddi, E., Gobat, R., et al. 2010, ApJL, 715, L6

Oser, L., Naab, T., Ostriker, J. P., \& Johansson, P. H. 2012, ApJ, 744, 63

Peng, C. Y., Ho, L. C., Impey, C. D., \& Rix, H.-W. 2002, AJ, 124, 266

Peng, Y.-j., Lilly, S. J., Renzini, A., \& Carollo, M. 2012, ApJ, 757, 4

Peng, Y., Lilly, S. J., Kovač, K., et al. 2010, ApJ, 721, 193

Poggianti, B. M., Calvi, R., Bindoni, D., et al. 2013, ApJ, 762, 77

Pozzetti, L., Bolzonella, M., Zucca, E., et al. 2010, A\&A, 523, A13

Rhodes, J. D., Massey, R. J., Albert, J., et al. 2007, ApJS, 172, 203

Sanders, D. B., Salvato, M., Aussel, H., et al. 2007, ApJS, 172, 86

Saracco, P., Longhetti, M., \& Gargiulo, A. 2010, MNRAS, 408, L21

Saracco, P., Longhetti, M., \& Gargiulo, A. 2011, MNRAS, 412, 2707

Sargent, M. T., Carollo, C. M., Lilly, S. J., et al. 2007, ApJS, 172, 434

Scarlata, C., Carollo, C. M., Lilly, S., et al. 2007a, ApJS, 172, 406

Scarlata, C., Carollo, C. M., Lilly, S. J., et al. 2007b, ApJS, 172, 494

Shankar, F., \& Bernardi, M. 2009, MNRAS, 396, L76

Shankar, F., Marulli, F., Bernardi, M., et al. 2013, MNRAS, 428, 109

Schechter, P. 1976, ApJ, 203, 297

Schlegel, D. J., Finkbeiner, D. P., \& Davis, M. 1998, ApJ, 500, 525

Scoville, N., Abraham, R. G., Aussel, H., et al. 2007, ApJS, 172, 38

Scranton, R., Johnston, D., Dodelson, S., et al. 2002, ApJ, 579, 48

Sérsic, J. L. 1963, BAAA, 6, 41

Szomoru, D., Franx, M., Bouwens, R. J., et al. 2011, ApJL, 735, L22

Taylor, E. N., Franx, M., Brinchmann, J., van der Wel, A., \& van Dokkum, P. G. 2010, ApJ, 722, 1

Toft, S., van Dokkum, P., Franx, M., et al. 2007, ApJ, 671, 285

Trenti, M., \& Stiavelli, M. 2008, ApJ, 676, 767

Trujillo, I., Conselice, C. J., Bundy, K., et al. 2007, MNRAS, 382, 109

Valentinuzzi, T., Poggianti, B. M., Saglia, R. P., et al. 2010, ApJL, 721, L19

van de Sande, J., Kriek, M., Franx, M., et al. 2013, ApJ, 771, 85

van der Wel, A., Bell, E. F., van den Bosch, F. C., Gallazzi, A., \& Rix, H.-W. 2009, ApJ, 698, 1232

van Dokkum, P. G., Franx, M., Kriek, M., et al. 2008, ApJL, 677, L5

Whitaker, K. E., Labbé, I., van Dokkum, P. G., et al. 2011, ApJ, 735, 86

Wijesinghe, D. B., da Cunha, E., Hopkins, A. M., et al. 2011, MNRAS, 415, 1002

Williams, R. J., Quadri, R. F., Franx, M., van Dokkum, P., \& Labbé, I. 2009, ApJ, 691,1879

York, D. G., Adelman, J., Anderson, J. E., Jr., et al. 2007, AJ, 120, 1579 